\title{
Completeness theorems for reactive modal logics
}

\section{Dov Gabbay}

Published online: 28 September 2012

(c) Springer Science+Business Media B.V. 2012

Keywords Modal logic. Temporal logic

Mathematics Subject Classifications (2010) 03 B $44 \cdot 03 B 45$

\section{Overview}

This paper gives completeness theorems for some basic reactive Kripke models and semantics. This section will

1. Introduce reactivity

2. Discuss and compare the kind of Kripke semantics we get with reactivity

3. Explain the challenges in obtaining completeness theorems

\subsection{Fibring and reactivity}

Our starting point is an ordinary Kripke model for modal logic. This has the form $\mathbf{m}=(S, R, a)$ where $S$ is a nonempty set of worlds, $a \in S$ is the initial (actual) world and $R \subseteq S \times S$ is the accessibility relation. The model also has an assignment $h$, giving for each atomic $q$ a subset $h(q) \subseteq S$.

We shall focus on $R$.

D. Gabbay

Bar-Ilan University, Ramat-Gan, Israel

D. Gabbay $(\bowtie)$

Department of Computer Science, King's College London, London, UK

e-mail: dov.gabbay@kcl.ac.uk

D. Gabbay

University of Luxembourg, Luxembourg City, Luxembourg 
Let us look at how the modality $\diamond$ is evaluated at a point $t \in S$. This is done by clause $(* 1)$

(*1) $t \vDash \diamond A$ iff $\exists s(t R s$ and $s \vDash A)$.

Let us write $(* 1)$ slightly differently, stressing which model we are using

(*2) $t \vDash \diamond A$ in the model $\mathbf{m}=(S, R, a)$ iff for some $s \in S$ such that $t R s$ we have $s \vDash A$ in $\mathbf{m}$.

Clause $(* 2)$ can be changed to a fibring clause. Suppose we associate with every $s \in$ $S$, a new Kripke model $\mathbf{m}_{s}=\left(S_{s}, R_{s}, s\right)$ and we modify $(* 2)$ to be $(* 3)$ for a fibred modality $\diamond_{F}$ (see [7]).

(*3) $\quad t \vDash \diamond_{F} A$ in $\mathbf{m}$ iff for some $s \in S$ such that $t R s$, we have $s \vDash A$ in $\mathbf{m}_{s}$.

Figure 1 describes the situation.

We switch evaluation at $s$ from the model $\mathbf{m}$ to the model $\mathbf{m}_{s}$.

In a way this is a kind of multimodal logic with models of the form $\mathbf{n}=\left(S, R_{S}, s \in\right.$ $S$ ) and the jump operators $J_{s}, s \in S$ and the modality $\diamond$. Evaluation is two dimensional as follows:

(*4) $\quad t \vDash_{s} \diamond A$ iff for some $u, t R_{s} u$ and $u \vDash_{s} A$ $t \vDash_{s} J_{u} A$ iff $t \vDash_{u} A$

According to $(* 4)$ the modality of $(* 3)$ can be written as

(*5) $\quad t \vDash_{s} \diamond_{F} A$ iff for some $u, t R_{s} u$ and $u \vDash_{s} J_{u} A$.

Note that public announcement logic (see references [1, 15]), reference [1] contains material on reactive public announcement logic is an example of this kind of operator. It has modalities of the form $\diamond_{A}$, where $A$ is a wff such that $t \vDash A$ holds. The truth condition is:

(*6) $t \vDash \diamond_{A} B$ in $(S, R)$ iff $t \vDash B$ in $\left(S_{A}, R\right)$, where $S_{A}=\{x \mid x \vDash A\}$.

The question to ask now is how does $\mathbf{m}_{s}$ relate to $\mathbf{m}$ ?

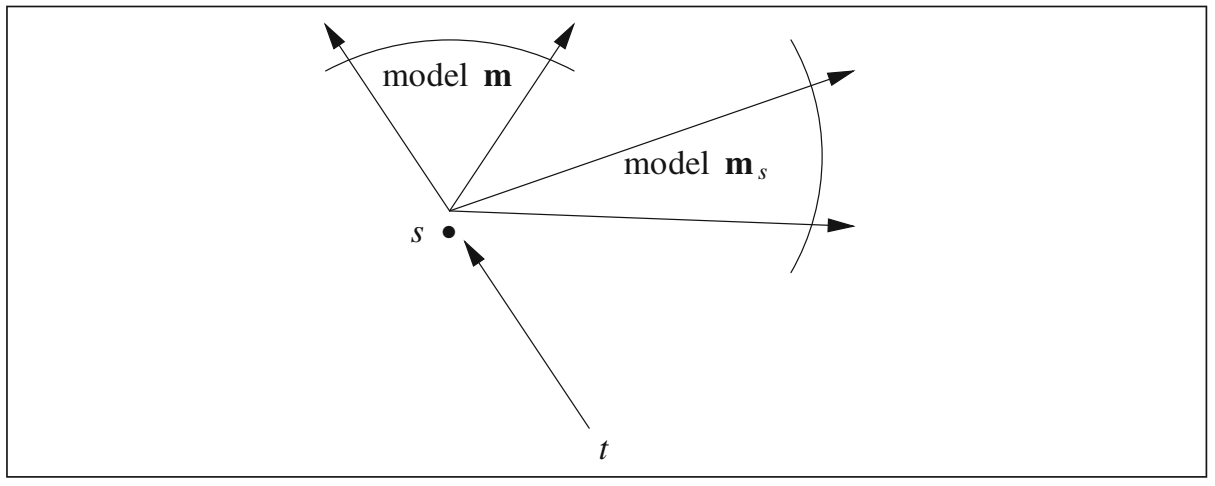

Fig. 1 Evaluation of modality at a point 
In reactive logic, we focus on $R_{S}$ being a variation of $R$, obtained by switching on and off accessibility connections in $R$. If we denote $(x, y) \in R$ by $x \rightarrow y$ then the basic reactive unit is the double arrow

$$
(x \rightarrow y) \rightarrow(u \rightarrow v) .
$$

The reading of the double arrow is as follows. If you traverse the connection $x \rightarrow y$, then send a signal and switch (on or off) the connection $u \rightarrow v$. Using a family $\mathbb{R}$ of double arrows, we can move from one $R_{s}$ to another $R_{s^{\prime}}$.

We now explain how reactivity can work in the next subsection.

\subsection{Reactive Kripke models}

Reactive Kripke models were introduced in 2004 [3] and an expanded version published in [5].

There are various options for kinds of reactivity

Example 1.1 (Reactive Kripke models) Consider the Kripke model of Fig. 2. For the moment ignore the double headed arrow in the diagram. If $R$ denotes the accessibility relation then we have in this model $a R c, a R b, c R d, b R d, d R e$. Let us try and evaluate $a \vDash \diamond \diamond \square \perp$.

For this to hold at $a$ we need a path $a R x, x R y$ and $y \vDash \square \perp$. Such a path is not available at this model.

Now let us look at the double arrow from the $\operatorname{arc}(a, c)$ to the $\operatorname{arc}(d, e)$. This double arrow is decorated with the minus sign. We read its meaning as saying disconnect the accessibility $d R e$ if you pass through the arc $(a, c)$. Thus if we get to $d$ through the path $a R b \wedge b R d$ then $d R e$ holds. However, if we get to $d$ through the path $a R c \wedge$

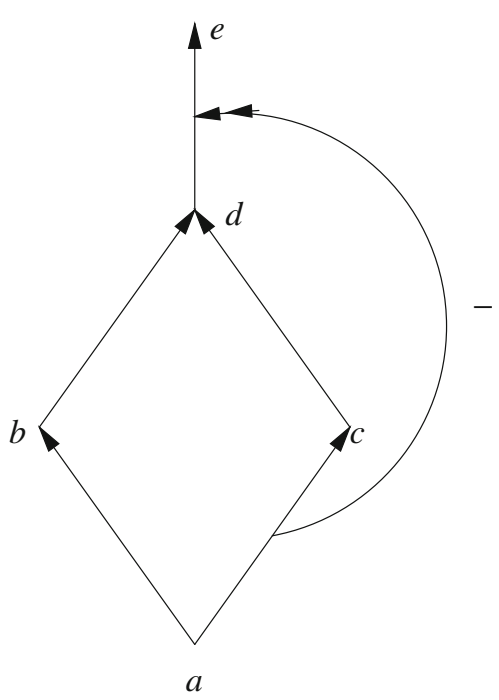

Fig. 2 A reactive Kripke model 
$c R d$, then by the time we get to $d, d R e$ does not hold. So in the reactive model of Fig. 2, where the double arrow is active we have that $a \vDash \diamond \diamond \square \perp$ holds because of the path $a R c \wedge c R d$.

Thus in a reactive Kripke model the double arrows change the models.

Figure 3 shows what we see at each point depending on the paths. In fact the Kripke model of Fig. 3 is an ordinary Kripke model where the nodes are paths. We must remember to give the same assignment to any atom $x$ at points $a b d$ and $a c d$. That is why we encircled them in the Figure.

We have all kinds of possibilities here, for the double arrow. See Figs. 4 and 5

1. Double arrow to an arc can switch it off (or on), i.e., disconnect (or connect) it. If decorated with '-' then it switches the arc to off if it is on and leaves it as off if it is off. If decorated with ' + ' then it switches the arc on if it is off and leaves it on if it is on. If not decorated at all then it switches it on if it is off and off if on, this is called switch reactivity.

2. Double arrow to a point can delete it or can tell us to ignore it or tell us to skip it.

So for example in Fig. 4, if point $d$ is ignored then at point $c$ the point $d$ is ignored and so

$$
a \vDash \diamond \square \perp
$$

holds because of $c$. But we still have

$$
a \vDash \diamond \diamond \diamond \square \perp
$$

because we can still pass through $d$ to get to $e$.

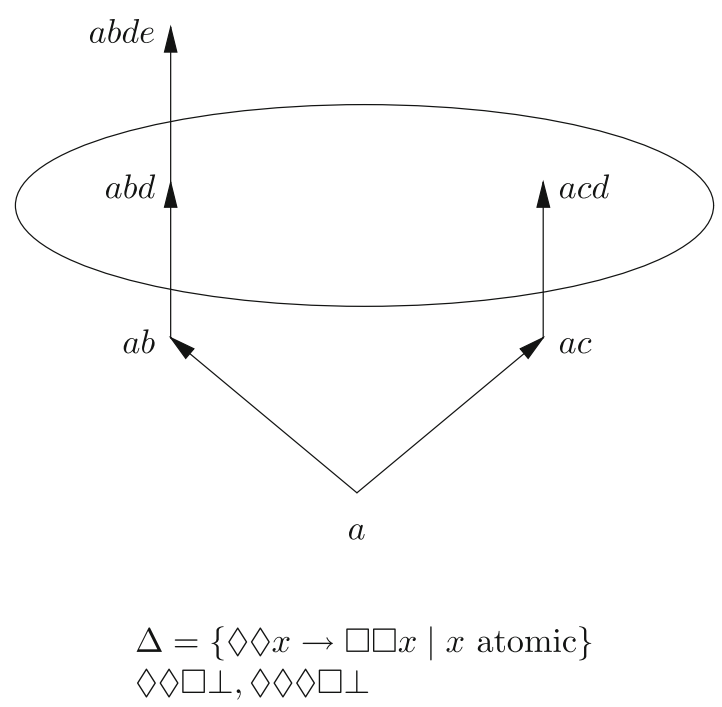

Fig. 3 A Kripke model with nodes as paths 


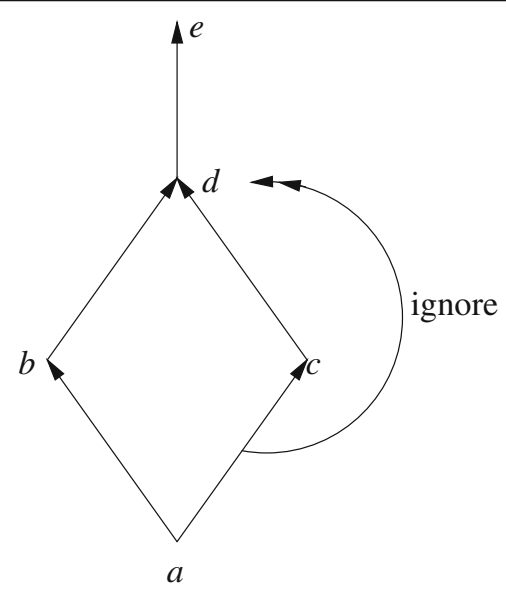

Ignore $d$

$$
\begin{aligned}
& \vDash \diamond \square \perp \\
& \vDash \diamond \diamond \diamond \square \perp
\end{aligned}
$$

Various options for making reactive using $\rightarrow$.

Fig. 4 The use of double arrows

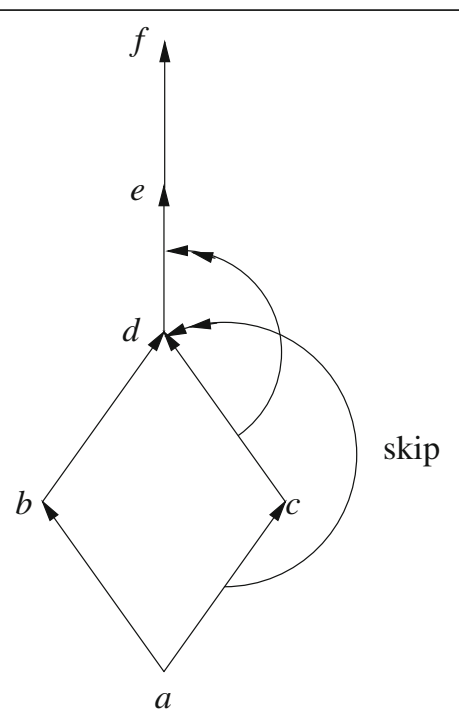

Skip $d$ but do go through the arc $(c, d)$ to get to $e$.

So at $c$ we have $c \vDash \diamond e$ even though we may have $d \vDash \neg e$.

Fig. 5 Reactive skip 
If we skip $d$ then at $c$ we see $e$ directly. If we delete $d$ then both $d$ and $e$ are deleted from the model, because $e$ is now unreachable from point $a$.

Theorem 1.2 There exist mono-modal logics which are complete for a class of reactive Kripke frames but are not complete for any class of ordinary Kripke or neighbourhood frames, see [5], and [4, Section 1].

Example 1.3 (Sample application: deontic logic) See [6, 14]. We model contrary to duty obligations using reactive Kripke models. See Fig. 6.

There should be no fence (i.e., go to $b$ ). But if there is a fence (i.e., you go to $c$ and because of that only worlds with fence are accessible), then it must be white (do not go to $e$, go to $d$ ).

Example 1.4 (Sample application: reactive grammars) See [10].

We make a context free grammar reactive by allowing the system to switch rules on and off as it progresses.

Figure 7 is such an example.

Theorem 1.5 Reactive context-free grammar can generate $\left\{a^{n} b^{n} c^{n} \mid n=1,2,3, \ldots\right\}$, which ordinary CFG cannot.

Example 1.6 (Sample application: reactive automata) See [2].

An automaton is a state transitions system responding to input. We can therefore change the automaton table as we go along. This will give us a reactive automaton. See Fig. 8.

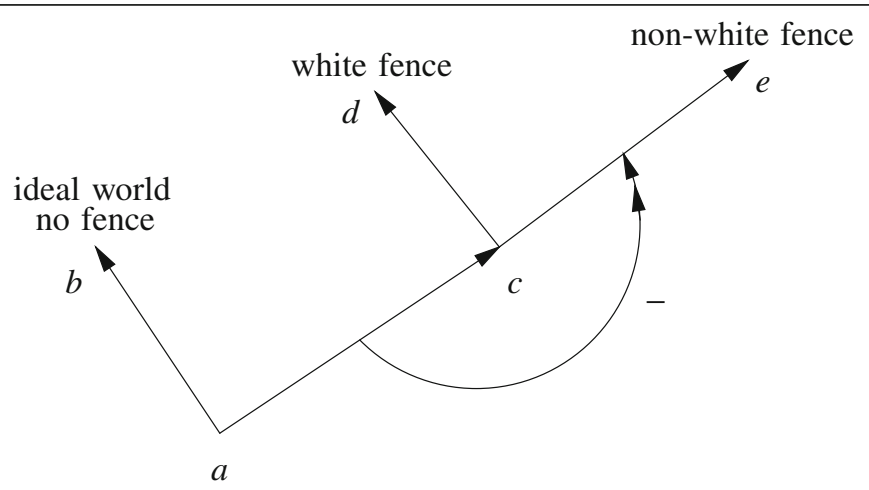

- There should be no fence

- If there is a fence it should be white

Fig. 6 Modelling contrary to duty obligations 
Reactive context-free grammar rules. (A rule contains a rewrite together with instructions which rules to activate and which to deactivate)

1. $Y \rightarrow \alpha X \beta$

activate 1 and deactivate 2

2. $X \rightarrow \gamma Z \delta$

Activate 2 and 3 and deactivate 1 .

3. $Z \rightarrow a Y$

no change

4. $Y \rightarrow \varnothing$

deactivate 4 , otherwise no change.

Fig. 7 Reactive grammar

Every reactive automaton is equivalent to an ordinary automaton recognising the same set of words. So we do not gain in expressive power by going reactive (as we do in the case of reactive context free grammars) but we do gain simplicity.

Theorem 1.7 Let $\mathcal{A}$ be an automaton (deterministic or non-deterministic) with $k=$ $\prod_{i=1}^{n} k_{i}$ states. Then there exists an equivalent reactive automaton $\mathcal{A}^{*}$ (deterministic or non-deterministic, resp) with $k^{*}=\sum_{i=1}^{n} k_{i}$ states.

Example 1.8 (Sample application: networks) See [9, 12, 13]. Figure 9 is an example of a network. It can be argumentation network, neural network, transportation network, predator-prey biological network and many more.

Here the double arrows have their own meaning in each network and their availability greatly enhances the expressive power of such networks.

Example 1.9 (Sample application: reactive proof theory) Figure 10 is an example of a reactive proof rule.

Rule $\mathbf{R}_{n}$ says that if $A_{1}^{n}, \ldots, A_{k(n)}^{n}$ are available, then we can infer $B_{n}$ but we need to activate Rules $D_{i}^{n}$ and deactivate Rules $E_{j}^{n}$.

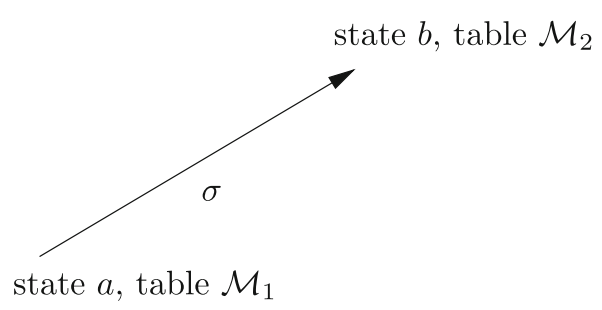

Fig. 8 Reactive automation 


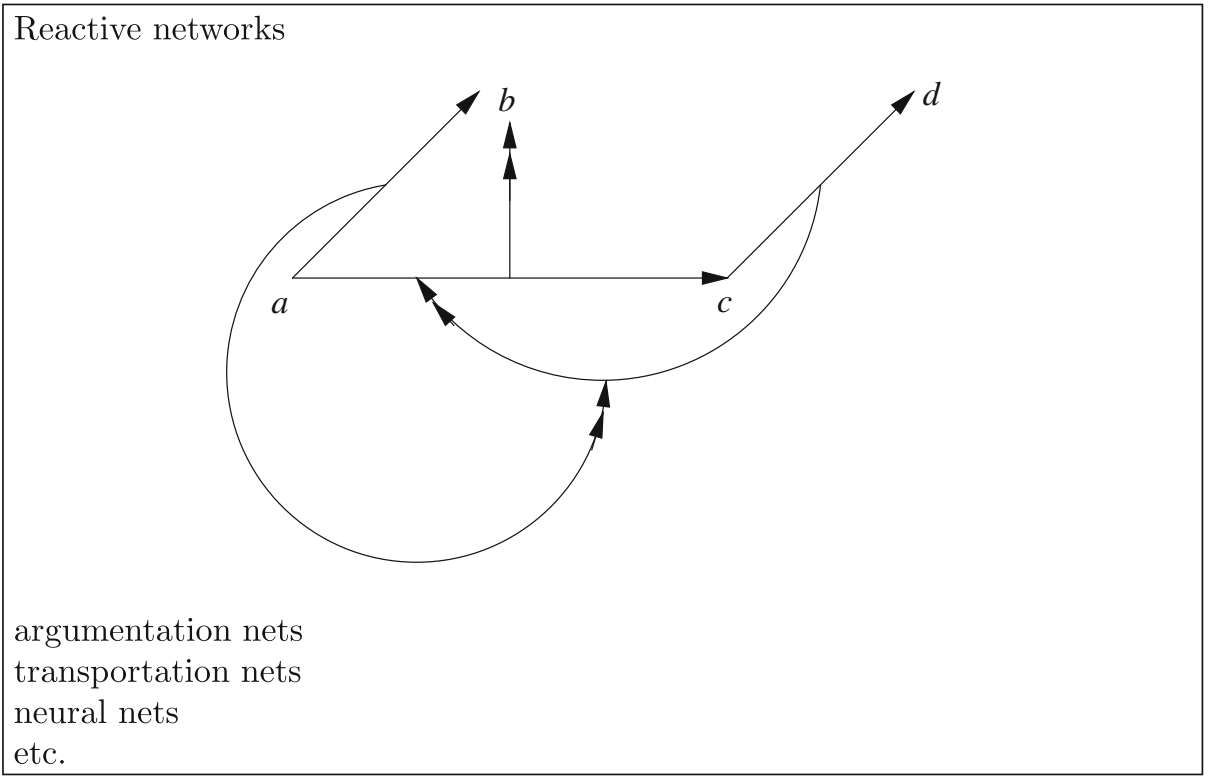

Fig. 9 Networks

For example Rule $\mathbf{R}_{n}$ can deactivate itself after use, as in linear logic. For reactive Tableaux, see $[4,8]$.

Theorem 1.10 See [11]. Every reactive Kripke model is equivalent to an ordinary Kripke model $(S, R, \equiv)$ with an additional equivalence relation $\equiv$ satisfying

$$
\neg \exists x y z(x R y \wedge x R z \wedge y \equiv z)
$$

and vice versa.

Figures 11, 12, 13 and 14 illustrate the need for the condition of the theorem. If $x \equiv y$ in Fig. 11, we need different paths leading to $x$ and $y$ so that we can fold the

\section{Reactive proof theory}

Rule $\mathbf{R}_{n}$

- $\frac{A_{1}^{n} \ldots A_{k(n)}^{n}}{B_{n}}$

- activate rules $D_{1}^{n}, \ldots, D_{m(n)}^{n}$

- deactivate rules $E_{1}^{n}, \ldots, E_{l(n)}^{n}$

Example: Goal directed proof theory deactivate rule once used!

Fig. 10 Reactive proof theory 


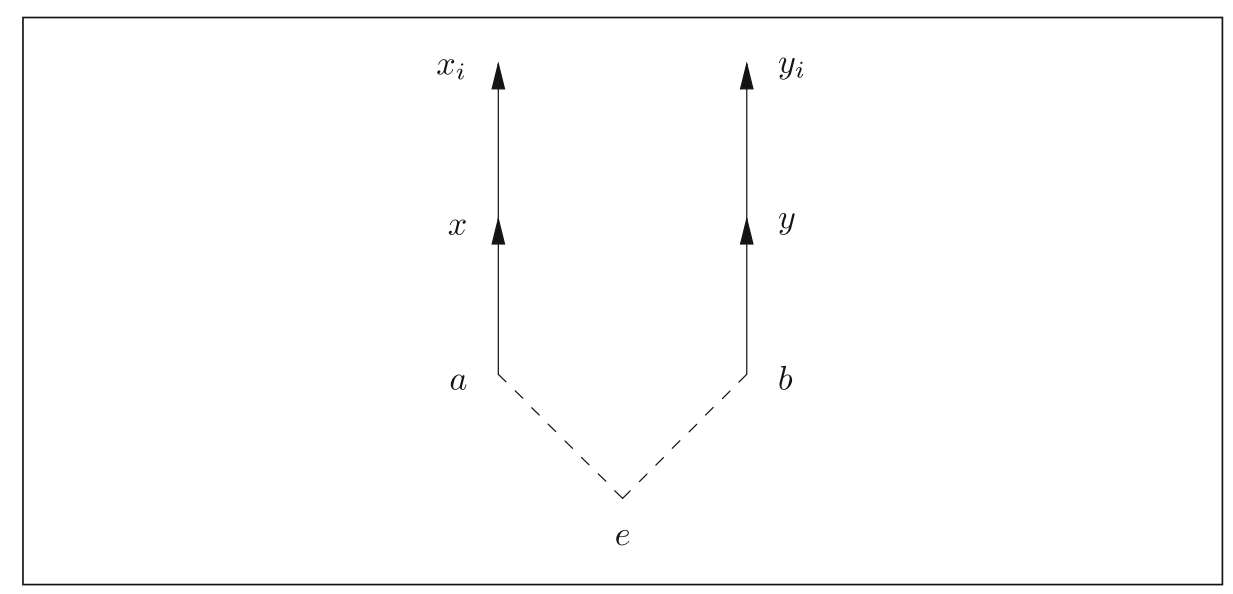

Fig. 11 Illustrating conditions for Theorem 1.10

model and make $x=y=z$, and then one path (leading to $x$ ) will see $x_{i}$ as accessible and the other path leading to $y$ will see the $y_{i}$ as accessible.

We get Fig. 12.

The situation in Fig. 13 does not allow us to have different paths to $z=x=y$ if we fold $x$ and $y$ together. All we can get is Fig. 14 .

\subsection{Ternary relations}

This section shows that reactive models can simulate a ternary relation and thus relevance logics and conditionals $A \Rightarrow B$ can be translated into a reactive modality $\square$. $A \Rightarrow B=\operatorname{def} \square(a \Rightarrow \square B)$. This also means that the classical modal reading of

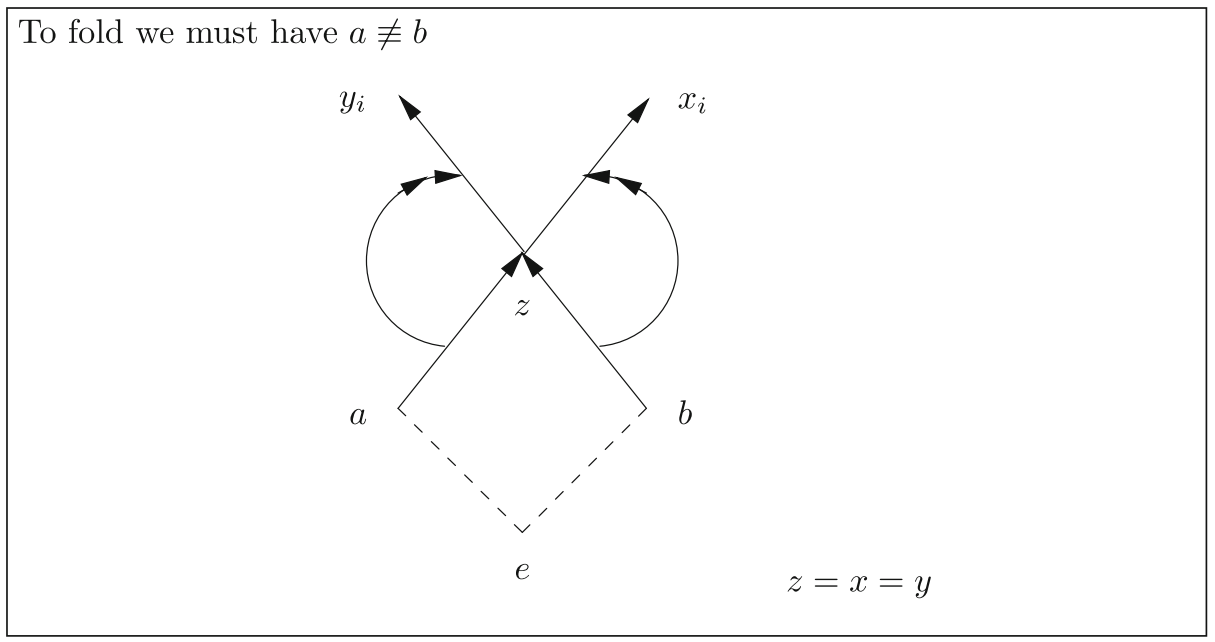

Fig. 12 What we need to fold 
We cannot fold

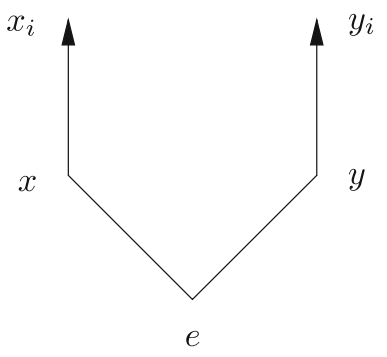

because we need different paths.

Fig. 13 We cannot fold

$t \vDash A \rightarrow B$ as $t \vDash A$ implies $t \vDash B$ is too poor to bring out the reachness of reactive Kripke models and we need something $A \Rightarrow B$ reading first evaluate $A$ and then evaluate $B$.

So $t \vDash A \Rightarrow B$ iff whenever we evaluate $t \vDash A$ in the model and the model changes as a result of the reactivity and we get a new model, then $t \vDash B$ holds in the new model.

Example 1.11 (Reduction of three place relation to reactive binary relation) This example shows how relevance logics, whose semantics requires a three place relation $\rho(x, y, z)$ can be simulated using a reactive binary relation $\mathbb{R}$.

We have for relevance $A \Rightarrow B$ the following truth condition

$$
x \vDash A \Rightarrow B \text { iff } \forall y z(\rho(x, y, z) \text { and } y \vDash A \text { imply } z \vDash B)
$$

The idea is very simple. Consider $\rho(x, y, z)$ as a binary relation between the pair $(x, y)$ and the points $z$. This we can represent in Fig. 15

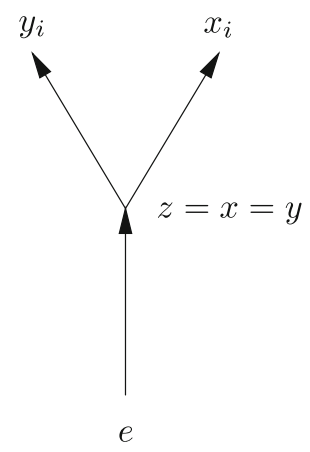

Fig. 14 Reducing Fig. 13 


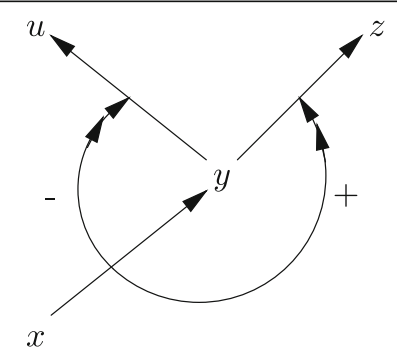

Fig. 15 Representing a ternary relation

In Fig. 15, we know that $(x, y)$ is related to $z$ because as we go from $x$ to $y$, we activate the connection $y \rightarrow z$. We also know that $(x, y)$ is not related to $u$ because as we go from $x$ to $y$ we cancel the connection $y \rightarrow u$.

Assume $\rho\left(x, y, z_{i}\right)$ holds but $\rho\left(x, y, v_{j}\right)$ does not. Let $\rightarrow+$ be an activating arrow from the $\operatorname{arc} x \rightarrow y$ to all the $z_{i}$ and $\rightarrow-$ be a deactivating arrow from the pair $x \rightarrow y$ to all the $v_{j}$.

The model is written as $(S, \mathbb{R}, \mathbf{a})$, where $\mathbb{R}$ is now a relation containing arrows $x \rightarrow y \quad($ written $(x, y) \in \mathbb{R})$, and double arrows $(x \rightarrow y) \rightarrow^{ \pm}(u, v)$, written as $+((x, y),(u, v)) \in \mathbb{R}$ or $-((x, y),(u, v)) \in \mathbb{R}$.

$\mathbf{a}$ is a $\{0,1\}$ function on arcs giving the active arcs and $\mathbf{a}_{(x, y)}$ is updated from $\mathbf{a}$ and $\mathbb{R}$ according to the $\pm((x, y)(u, r)) \in \mathbb{R}$.

$\mathbf{a}_{(t, s)}$ is modified from $\mathbf{a}$ as follows:

- $\mathbf{a}_{(t, s)}(\beta)=1-\mathbf{a}(\beta)$, if $[+((t, s), \beta) \in \mathbb{R}$ and $\mathbf{a}(\beta)=0]$ or $[-((t, s), \beta) \in \mathbb{R}$ and $\mathbf{a}(\beta)=1]$.

- $\mathbf{a}_{(t, s)}(\beta)=\mathbf{a}(\beta)$ otherwise.

Thus when we pass from $x$ to $y$, we activate only those $z_{i}$ such as $\rho\left(x, y, z_{i}\right)$ holds.

Thus if $A \Rightarrow B$ denotes the binary relevance or conditional connective whose semantics involves the ternary relation $\rho(x, y, z)$ and $\square$ represents a reactive modality, then we translate

$$
A \Rightarrow B=\operatorname{def} . \square(A \rightarrow \square B)
$$

We expect that for every model $(S, \rho)$ of $\Rightarrow$ there exists a model $(S, \mathbb{R}, \mathbf{a})$ of $\square$, such that for every $A, B$ and $t \in S$ and $h$ we have

$$
t \vDash_{h} A \Rightarrow B \text { in }(S, \rho, h) \text { iff } t \vDash \square(A \Rightarrow \square B) \text { in }(S, \mathbb{R}, \mathbf{a}, h) .
$$

The translation of $\rho(x, y, z)$ suggested in Fig. 15 is not good enough. Consider the case of 2 points $x, y$ and assume we have exactly $\rho(x, x, x)$ and $\rho(x, y, y)$

Figure 16 shows our proposed implementation

From $x$ we go to $x$. We cancel all paths from $x$ to points $z$ such that $\neg \rho(x, x, z)$ and connect all paths to $z^{\prime}$ such that $\rho\left(x, x, z^{\prime}\right)$ holds. So after going from $x$ to $x$ we are now at $x$ again and we have no connection to $y$. 


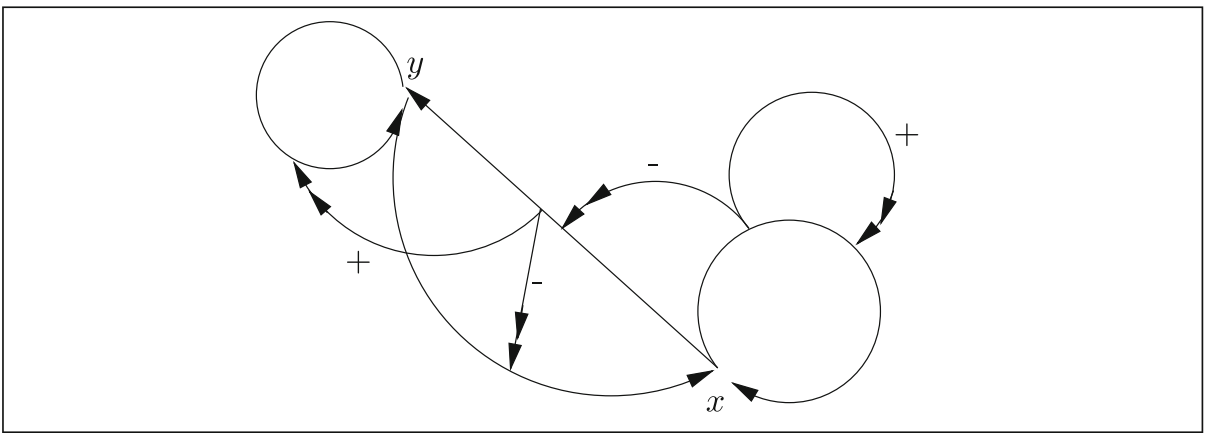

Fig. 16 Better representation of a ternary relation

So let us evaluate at $x$ the formula of relevance implication $A \Rightarrow(B \Rightarrow C)$.

$$
x \vDash A \Rightarrow(B \Rightarrow C) \text { iff } \forall z z^{\prime}\left(\left(\rho\left(x, z, z^{\prime}\right) \wedge z \vDash A \text { implies } z^{\prime} \vDash B \Rightarrow C\right)\right. \text {. }
$$

In our case we need to check the cases of $\rho(x, x, x)$ and $\rho(x, y, y)$. Figure 16 shows that in both cases the relation works OK, but we have to evaluate now $x \vDash B \Rightarrow C$ and $y \vDash B \Rightarrow C$ (assuming $x \vDash A$ and $y \vDash A$ hold).

But we cannot continue correctly because the diagram has changed. What we need is to go back to the initial position!

To implement $\rho(x, y, z)$, we need to do the following:

1. As we move from $x$ to $y$, we connect $y$ only to $\{z \mid \rho(x, y, z)\}$. Call this action as 'making sure that $\lambda z \rho(x, y, z)$ is correct for $(x, y)$ '.

2. If we continue now from $y$ to $z$ we do not want to activate the action described in (1) for $(y, z)$, i.e., we do not want at this stage to 'make sure that $\lambda w \rho(y, z, w)$ is correct for $(y, z)^{\text {', }}$, we just want to get to $z$. So in (1) while we are dealing with $\lambda z \rho(x, y, z)$, we deactivate all actions for $\lambda w \rho(y, z, w)$. This requires double arrows of order 2 .

3. As we actually pass through $y \rightarrow z$, we reactivate all actions for $\lambda w \rho(y, z, w)$.

We therefore propose to translate $\rho(x, y, z)$ as in Fig. 17.

In this figure, $\rho(x, y, z)$ and $\neg \rho(x, y, u)$ and $\rho(y, z, v)$ and $\neg \rho(y, z, w)$ hold.

Given a relation $\rho \subseteq S \times S \times S$, we simulate $(S, \rho)$ by a model $(S, \mathbb{R}, \mathbf{a})$ built as follows.

Let $\mathbf{a}(x, y)=1$ for all $(x, y) \in S \times S$. Define $\mathbb{R}$ as follows:

$(\sharp 1) \quad. S \times S \subseteq \mathbb{R}$

$(\sharp 2) \quad+.((x, y),(y, z)) \in \mathbb{R}$ if $\rho(x, y, z)$ holds, for all $x, y, z$

$(\sharp 3$.) $\quad-((x, y),(y, z)) \in \mathbb{R}$ if $\sim \rho(x, y, z)$ holds, for all $x, y, z$.

$(\sharp 4$.$) \quad if \rho(x, y, z)$ holds and $\pm \rho(y, z, w)$ holds, then by (2) above $\pm((y, z),(z, w) \in \mathbb{R}$ (respectively).

We add to $\mathbb{R}$ also $-((x, y),((y, z),(z, w))) \in \mathbb{R}$ and also $+((y, z),((y, z)$, $(z, w))) \in \mathbb{R}$. 


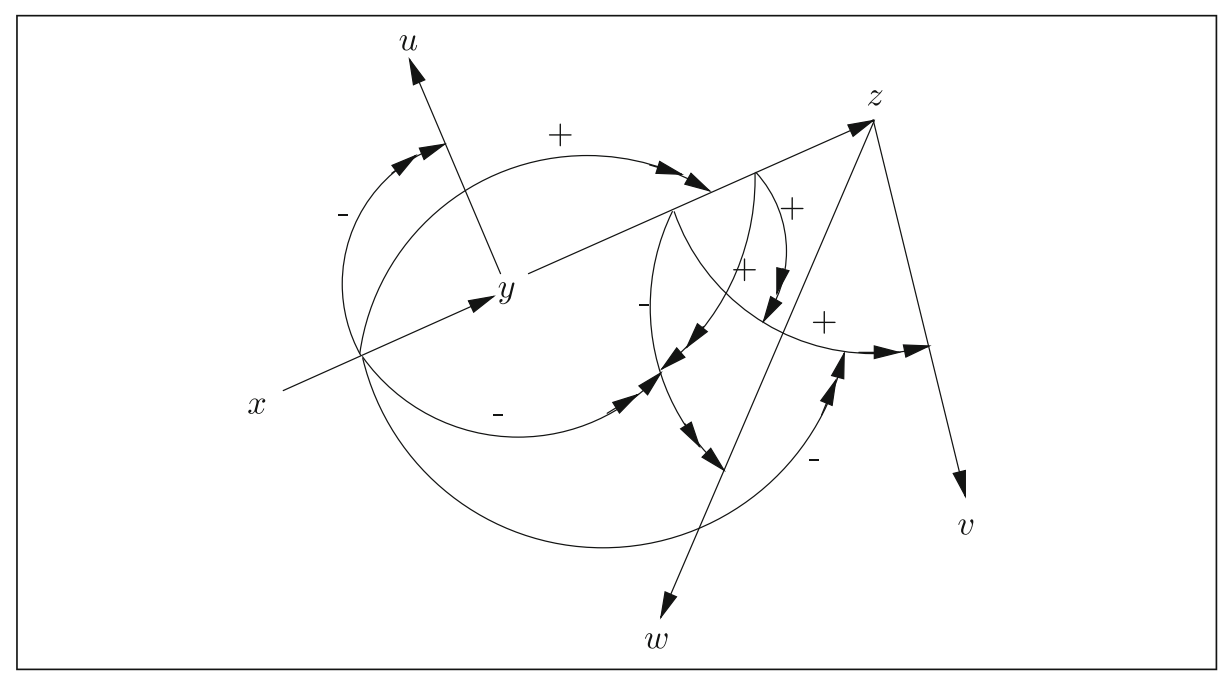

Fig. 17 Even better representation of a ternary relation

Now consider the relevance truth condition in a model $(S, \rho, t)$ with a ternary $\rho$ for the relevant $A \Rightarrow B$. It is

(*) $x \vDash A \Rightarrow B$ iff $\forall y z(\rho(x, y, z) \wedge y \vDash A$ imply $z \vDash B)$.

Consider now the evaluation of $\square(A \rightarrow \square B)$ at $x$ at a reactive model $(S, \mathbb{R}, \mathbf{a}, x)$

(**) $\quad x \vDash \square(A \rightarrow \square B)$ iff for all $y$ such that $(x, y) \in \mathbb{R}$ and $\mathbf{a}(x, y)=1$ (i.e., for all $\operatorname{arcs} x \rightarrow y$ which are active), we have $y \vDash A \rightarrow \square B$ holding at the model $\left(S, \mathbb{R}, \mathbf{a}_{(x, y)}, y\right)$ where $\mathbf{a}_{(x, y)}$ is the updated activeness function.

We continue:

(***) $\quad y \vdash A \rightarrow \square B$ iff whenever $y \vDash A$ then for all $z$ such that $(y, z) \in \mathbb{R}$ and is $\mathbf{a}_{(x, y)}$ active we have $z \vDash B$ in the model $\left(S, \mathbb{R}, \mathbf{a}_{(x, y, z)}, z\right)$.

To have both definitions $(*)$ and $(* *)$ and $(* * *)$ to be the same we need $\rho(x, y, z)$ to hold iff $\mathbf{a}_{(x, y)}(y, z)=1$.

Indeed, this is what Fig. 17 and items $(\sharp 1)-(\sharp 4)$ show.

So we have the following:

1. Given a reactive frame $(S, \mathbb{R}, \mathbf{a})$ the relation $\rho(x, y, z)$ defined by $\left(\mathbf{a}_{(x, y)}(y, z)=1\right.$ gives us a relevance frame $(S, \rho)$. We have for $A \Rightarrow B=\operatorname{def} \square(A \rightarrow \square B)$ that $x \vDash A \Rightarrow B$ iff $(* *)+(* * *)$ hold iff (*) holds for $\rho$.

2. We have also seen that given $(S, \rho)$, if we define $(S, \mathbb{R}, \mathbf{a})$ as in $(\sharp 1)-(\sharp 4)$, we can simulate $\rho$.

Thus the translation of $A \Rightarrow B$ as $\square(A \rightarrow \square B)$ is faithful.

The interesting aspect of this example is that axioms on $\Rightarrow$ become genuine axioms on the reactivity of a Kripke frame. 


\section{Introducing the logic $K R$, reactive modal $K$}

We have two options for defining the reactive counterpart of modal $\mathbf{K}$.

Option 1 Use only the modality which triggers the double arrows. Let us denote by $\varnothing A$ and its possibility by

$$
\mathbb{P} A=\neg \oslash \neg A .
$$

We have studied completeness theorems for such systems in [11].

Option 2 Study modal $\mathbf{K}$ with both $\oslash$ and $\mathbb{P}$ and the ordinary $\square$ and $\oslash$. We call this system KR. It has more expressive power and more applications, see [4, 14].

This paper deals with Option 2. The reactivity we used is switch reactivity.

\subsection{Introducing and discussing the reactive semantics}

Definition 2.1 (Reactive K model)

1. Let $S$ be a non-empty set. A reactive relation on $S$ has the form

$$
R=R_{1} \cup R_{2}
$$

where $R_{1} \subseteq S^{2}$ and $R_{2} \subseteq S^{2} \times S^{2}$ such that the following holds

$$
((x, y),(w, z)) \in R_{2} \rightarrow(x, y) \in R_{1} \wedge(w, z) \in R_{1} .
$$

(This means $R_{2} \subseteq R_{1} \times R_{1}$ ).

We can represent the elements of $R_{1}$ as ' $x \rightarrow y$ ' and the elements of $R_{2}$ as ' $(x \rightarrow y) \rightarrow(w \rightarrow z)$ '. The elements of $R_{1}$ are called arrows and those of $R_{2}$ are called double arrows.

2. A function $\mathbf{f}: R_{1} \mapsto\{0,1\}$ is called an activity function. When $\mathbf{f}(x, y)=1$ we say $x \rightarrow y$ is 'on' or is 'active' and we write $x \stackrel{+}{\rightarrow} y$. When $\mathbf{f}(x, y)=0$, we say $x \rightarrow y$ is 'off' or 'not active' and we write $x \stackrel{-}{\rightarrow} y$.

3. An assignment $h$ is a function giving each atomic $q$ a subset $h(q) \subseteq S$.

4. A model has the form $\mathbf{m}=\left(S, R_{1}, R_{2}, \mathbf{f}, a, h\right)$ where $a \in S$ is the initial world.

Definition 2.2 (Satisfaction) Our language contains the classical connectives $\neg, \wedge, \vee, \rightarrow, \top, \perp$ and the two modalities $\square$ and $\oslash$. We define satisfaction in a model as follows.

1. Let $\left(S, R_{1}, R_{2}, \mathbf{f}\right)$ be given. Let $x \stackrel{+}{\rightarrow} y$ be in $R_{1}$. This means that $(x, y) \in R_{1}$ and $\mathbf{f}(x, y)=1$. We define $\mathbf{f}[x, y]$ as follows:

$$
\mathbf{f}[x, y](u, v)=\left\{\begin{array}{l}
\mathbf{f}(u, v) \text { if }((x, y),(u, v)) \notin R_{2} \\
1-\mathbf{f}(u, v)) \text { if }((x, y),(u, v)) \in R_{2}
\end{array}\right.
$$

2. Given a model $\mathbf{m}=\left(S, R_{1}, R_{2}, \mathbf{f}, a, h\right)$ we define the notion of $\mathbf{m} \vDash A$ by induction as follows:

- $\quad \mathbf{m} \vDash q$ iff $a \in h(q)$, for $q$ atomic

- $\mathbf{m} \vDash A \wedge B$ iff $\mathbf{m} \vDash A$ and $\mathbf{m} \vDash B$

- $\mathbf{m} \vDash \neg A$ iff $\mathbf{m} \not \models A$ 
- Similarly for the other classical connectives

- $\quad \mathbf{m} \vDash \square A$ iff for all $y$ such that $(a, y) \in R_{1}$ we have $\mathbf{n}=\left(S, R_{1}, R_{2}, \mathbf{f}, y, h\right) \vDash A$

- $\mathbf{m} \vDash \oslash A$ iff for all $y$ such that $(a, y) \in R_{1}$ and $\mathbf{f}(a, y)=1$ we have $\mathbf{n}=$ $\left(S, R_{1}, R_{2}, \mathbf{f}[a, y], y,\right) \vDash A$.

3. The logic KR (reactive $\mathbf{K}$ ) is defined as the set of all wffs $A$ such that for all models $\mathbf{m}$ we have $\mathbf{m} \vDash A$.

4. Note that we have frames of the form $\left(S, R_{1}, R_{2}, \mathbf{f}\right)$. The logic is normal, as $a$ and $h$ can be chosen arbitrarily.

Remark 2.3 Note that the function $\mathbf{f}$ introduced in the previous Definition 2.1 actually defines another relation $R_{0}$ which is a subset of $R_{1}$. When $\mathbf{f}$ changes to $\mathbf{f}_{[x, y]}$, as in item 1 of Definition 2.2, then equivalently $R_{0}$ changes to $R_{0}[x, y]$. We use the notation with the function $\mathbf{f}$ to stress the fact that all reactivity changes arise from arrow connections from $R_{1}$.

We now address the task of axiomatising the logic KR. To be able to do that effectively, let us familiarise ourselves better with $\mathbf{K R}$ and its unique features.

Consider Fig. 18.

Let our starting point be $a$. As we traverse the arc $a \rightarrow b$, there are two possibilities.

1. We ignore all double arrows and what they do.

2. We take account of what the double arrows say and what they do.

Let Possibility 1 be formalised by $\diamond$ and let Possibility 2 be formalised by $\mathbb{P}$. So we can write: $a \vDash \Delta q$ to mean traverse from $a$ to the accessible world $b$ and ignore all double arrows and we can write $a \vDash \mathbb{P} q$ to mean traverse from $a$ to the accessible world $b$ and take account of the double arrows.

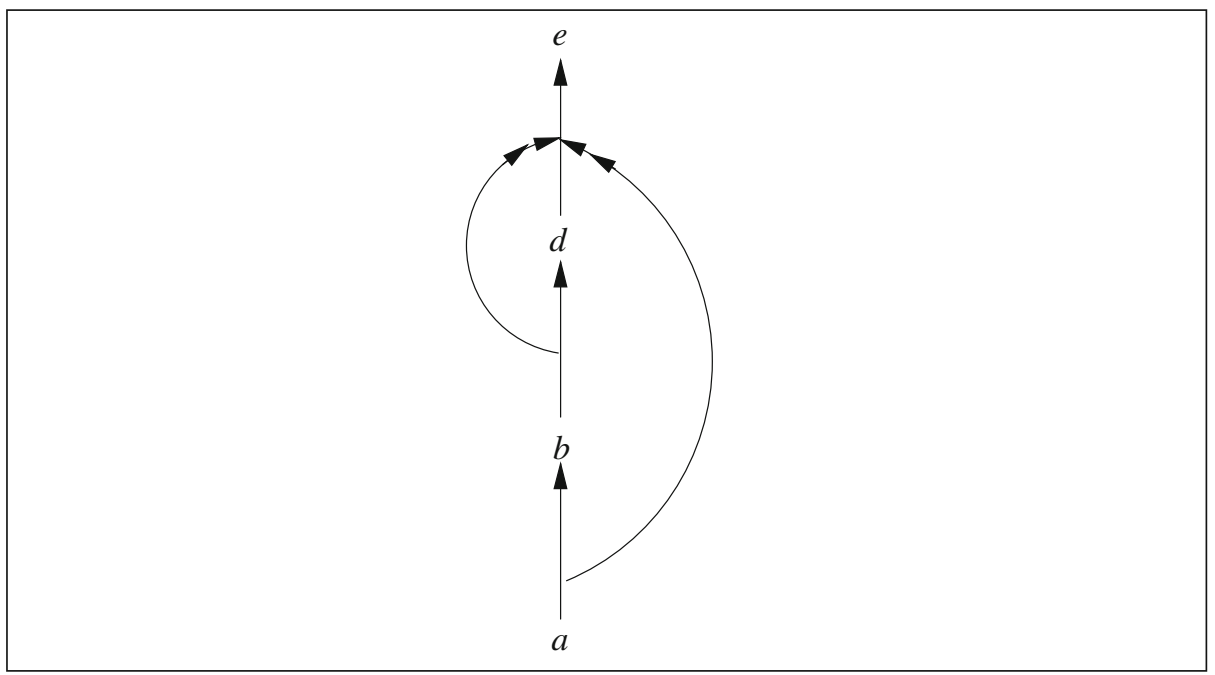

Fig. 18 Two double arrows hitting the same arc 
Consider now

$$
a \vDash \mathbb{P} \diamond \diamond \top
$$

Here we move from $a$ to $b$ and activate the double arrow which disconnects the arc $d \rightarrow e$. We check:

$$
b \vDash \diamond \diamond \top
$$

and then

$$
d \vDash \diamond \top
$$

Now the arc $d \rightarrow e$ is disconnected by a double arrow but as we are evaluating $\diamond$ at node $d$ and $\diamond$ ignores all double arrows and their effects, as far as $\diamond$ is concerned $d \rightarrow e$ is connected and hence $d \vDash \diamond \top$ holds and therefore $a \vDash \mathbb{P} \diamond \diamond \top$ also holds.

The situation is different with

$$
a \vDash \mathbb{P} \diamond \mathbb{P} \top
$$

Evaluating here takes us to $d \vDash \mathbb{P} T$, but $\mathbb{P}$ is affected and takes account of double arrows and so $d \vDash \mathbb{P} T$ does not hold and hence

$$
a \vDash \mathbb{P} \diamond \mathbb{P} \top
$$

does not hold.

Consider now

$$
a \vDash \mathbb{P P P} \top \text {. }
$$

We can go from $a$ to $e$ taking account of double arrows. Moving from $a$ to $b$ switches the connection $d \rightarrow e$ off but continuing from $b$ to $d$ switches it on again and therefore $d \vDash \mathbb{P} T$ holds.

Note that we cannot evaluate any $x \vDash \mathbb{P} q$, for arbitrary point $x$, without knowing how we got to $x$ and which double arrows we activated along the path.

So asking does $d \vDash ? \mathbb{P} T$ hold cannot guarantee an answer. We need to ask something like

$$
a \stackrel{\diamond}{\longrightarrow} b \stackrel{\mathbb{P}}{\longrightarrow} d \vDash ? \mathbb{P} \top
$$

or

$$
a \stackrel{\mathbb{P}}{\longrightarrow} b \stackrel{\diamond}{\longrightarrow} d \vDash ? \mathbb{P} \top
$$

or in general

$$
\left(x_{0} \stackrel{M_{1}}{\longrightarrow} x_{1} \stackrel{M_{2}}{\longrightarrow} x_{2} \rightarrow \ldots \stackrel{M_{n}}{\longrightarrow} x_{n}\right) \vDash ? A
$$

where $M_{i} \in\{\diamond, \mathbb{P}\}$.

Note that we need a notion of legitimate sequences. This notion will be formally defined in Section 2.2 below. Meanwhile, we understand it intuitively. The sequence

$$
a \stackrel{\mathbb{P}}{\longrightarrow} b \stackrel{\diamond}{\longrightarrow} d \stackrel{\mathbb{P}}{\longrightarrow} e
$$

is not legitimate because by the time we get to $d, d \rightarrow e$ is disconnected. So to get to $e$ from $d$ we need to use $\diamond$. 
We thus get a bimodal logic with two modalities $\diamond$ and $\mathbb{P}$ with evaluation of formulas depending on paths $\left(x_{0}, \ldots, x_{n}\right)$ but not ordinary paths. We also need to know how we traverse them. So our paths have the form $\mathbf{t}=\left(x_{0}, M_{1} x_{1}, M_{2} x_{2}, \ldots, M_{n} x_{n}\right)$, where $\mathbf{t}$ is a legitimate sequence.

Our task in this section is to find axioms and prove completeness for KR. We might have thought that it would be useful to consider KR as a bimodal logic with two accessibility relations $R_{\mathbb{P}}$ and $R_{\diamond}$, and see what properties they have. The problem is that the accessible worlds are the same for both relations, the difference being the way we traverse them. So really our most reasonable option is to consider a transformed Kripke model where the points/worlds are legitimate paths of the form

$$
\mathbf{t}=\left(a, M_{1} x_{1}, \ldots, M_{n} x_{n}\right)
$$

such that $a R_{1} x_{1} R_{1} x_{2}, \ldots, x_{n-1} R_{1} x_{n}$ holds.

Thus we start with

$$
\mathbf{m}=\left(S, R_{1}, R_{2}, a, h\right)
$$

for example $\left(S, R_{1}, R_{2}, a\right)$ is as in Fig. 18, and then transform it to a model

$$
\mathbf{m}^{*}=\left(S^{*}, R_{\diamond}, R_{\mathbb{P}},(a), h\right)
$$

where $S^{*}$ is the set of all legitimate sequences of the form $\mathbf{t}=\left(a, M_{1} x_{1}, \ldots, M_{n} x_{n}\right)$ where $a R_{1} x_{1} \wedge x_{1} R_{1} x_{2} \wedge, \ldots, \wedge x_{n-1} R_{1} x_{n}$.

We define $R_{\diamond}$ and $R_{\mathbb{P}}$ by

$$
\mathbf{t} R_{\diamond} \mathbf{t} *(M y), \text { when } x_{n} R_{1} y, \text { and } M \in\{\diamond, \mathbb{P}\}
$$

$\mathbf{t} R_{\mathbb{P}} \mathbf{t} *\left((\mathbb{P} y)\right.$, when $x_{n} R_{1} y$ and $\mathbf{t} *(\mathbb{P} y)$ is legitimate.

where $*$ is concatenation of sequences.

Let $\mathbf{t} \vDash q$ iff $x_{n} \vDash q$, for $q$ atomic.

Note that $R_{1}$ and $R_{2}$ are replaced by $R_{\diamond}$ and $R_{\mathbb{P}} . R_{1}$ is used directly in the definition of the sequences $\mathbf{t}$ which go into $S^{*}$ and $R_{2}$ is used in the notion of legitimate sequences.

So $\mathbf{m}$ is replaced by its transformed model $\mathbf{m}^{*}$, see Fig. 19.

If we do that we can get a completeness theorem after some hard work. We are facing three formal tasks:

Task 1: Given a bimodal semantic model $\mathbf{m}^{*}$ with two binary relation $R^{\prime}$ and $R^{\prime \prime}$, and with an equivalence relation $\equiv$, such as the one in Fig. 19, can we identify whether it comes from a KR model $\mathbf{m}$ such as the one in Fig. 18? In other words, can we identify when a bimodal model $\mathbf{n}^{*}$ with an equivalence relation $\equiv$ is actually a transformed version of another $\mathbf{K R}$ model $\mathbf{n}$ ?

Task 2: Axiomatise the logic $\mathbf{K}$.

Task 3: We need to systematically find corresponding axioms for various conditions on the $\mathbf{K R}$ relation $R_{1}$. This Task we postpone to a future paper.

Obviously the transformed models will play a part, but we note that the transformed models may not be in the same class as the original model. The lucky aspect for the case of $\mathbf{K R}$ is that such models $\mathbf{m}^{*}$ are also $\mathbf{K R}$ models because $\mathbf{K R}$ has no special requirements on the worlds or the relations. 


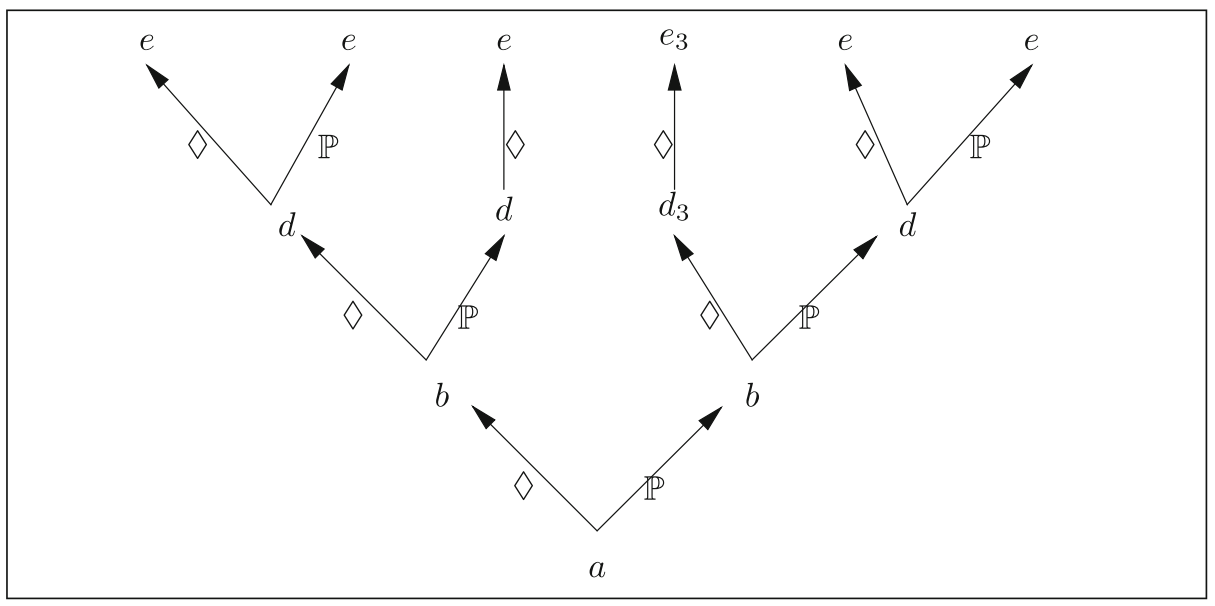

Fig. 19 Figure 18 transformed

The unlucky or difficult aspect of using the transformed models as tools is that we need to uniquely identify paths, and to do that we need nominals or second order propositional quantifiers or in short we need more expressive power at our service and such power is not available at KR. So our strategy is to use technical devices which eliminate the need for nominals.

So much for KR. If we look at Fig. 18 we observe that the model is linear. If we were to insist on semantics which requires that $\left(S, R_{1}\right)$ be linear then looking at the model $\mathbf{m}^{*}$ of all legitimate paths gives us a non-linear model. This is not good. We are getting a model which is not in the correct linear semantics. So how do we axiomatise the linear case? The answer is that I don't know yet. For the purpose of many applications such as modelling contrary to duties, $\mathbf{K R}$ is sufficient, see [14]. We postpone the general theory of reactive modal semantics for dedicated future papers.

We now give you an idea of what kind of axioms we need for KR.

We first note that both $\square$ and $\oslash$ (the duals of $\diamond$ and $\mathbb{P}$ ) are $\mathbf{K}$ modalities, satisfying the interaction axiom (for $A$ not containing any $\oslash$ ).

$$
\mathbb{P} A \rightarrow \diamond A
$$

To explain what additional axioms we need, consider again Fig. 18 which describes $S$ and compare with Fig. 19 which describes $S^{*}$.

Figure 19 is a tree and so each node gives the sequence leading up to it. The indicies on some nodes are there to help us talk about them. The actual sequences have no indices. So $\left(a \mathbb{P} b \diamond d_{3} \diamond e_{3}\right)$ is really the legitimate sequence $(a \mathbb{P} b \diamond d \diamond d)$.

Since the sequence $\left(a \mathbb{P} b \diamond d \mathbb{P} e\right.$ ) is not legitimate, the point $d_{3}$ is $\diamond$ connected only to $e_{3}$ and is not $\mathbb{P}$ connected to $e$.

\footnotetext{
${ }^{1}$ This figure describes a bimodal logic model with a set of possible worlds $S *$ and with two accessibility relations, as indicated, and an additional equivalence relation $\equiv$ on worlds. The equivalence relation can be read from the fact that we named in the figure several nodes by the same letter. When node $x$ is equivalent to node $y$, we are committed to give the same truth values to atoms at these nodes.
} 
We now show you how to get an axiom. The axioms which we get allow us to retrieve the double arrow from the graph. Since

1. $(a \diamond b \diamond d \mathbb{P} e)$ is legitimate

and

2. $(a \mathbb{P} b \diamond d \mathbb{P} e)$ is not legitimate

we know that there must be a double arrow

$$
(a \rightarrow b) \rightarrow(d \rightarrow e) .
$$

Similarly, we identify a double arrow from $(b \rightarrow d) \rightarrow(d \rightarrow e)$ since

3. $(a \diamond b \diamond d \mathbb{P} e)$ is legitimate

and

4. $(a \diamond b \mathbb{P} d \mathbb{P} e)$ is not legitimate.

From this we know that if we use both double arrows, the move $d \stackrel{\mathbb{P}}{\longrightarrow} e$ becomes possible. So

5. $(a \mathbb{P} b \mathbb{P} d \mathbb{P} e)$ is legitimate.

The axiom we get in this case is

Axiom: $\quad(1) \wedge(2) \wedge(3) \wedge(4) \rightarrow(5)$.

This axiom is not written in the modal language of KR. To express it in KR we need extra technical manipulation. If we have nominals, i.e., we have names for worlds, say $\bar{a}$ names $a, \bar{b}$ names $b, \bar{d}$ names $d$ and $\bar{e}$ names $e$, i.e., we have

$$
y \vDash \bar{x} \text { iff } y=x,
$$

then we can express the following in the logic $\mathbf{K R}+$ nominals:

(*): " $\left(a M_{1} x_{1} M_{2} x_{2} \ldots M_{n} x_{n}\right)$ is legitimate"

by writing $a \vDash \alpha_{*}$ where

$$
\alpha_{*}=M_{1}\left(\overline { x } _ { 1 } \wedge M _ { 2 } \left(\bar{x}_{2} \wedge \ldots \wedge\left(M_{n-1}\left(\bar{x}_{n-1} \wedge M_{n} \bar{x}_{n}\right) \ldots\right)\right.\right.
$$

Thus our axiom for Fig. 18 is

$$
\alpha_{1} \wedge \alpha_{2} \wedge \alpha_{3} \wedge \alpha_{4} \rightarrow \alpha_{5}
$$

In general if $\mathbf{t} *(\mathbb{P} y)$ is legitimate (not legitimate) and $\mathbf{t}_{1}$ and $\mathbf{t}_{2}$ are obtained by changing a single $\diamond$ into $\mathbb{P}$ at each place $n_{1}$ and $n_{2}$ respectively and $\mathbf{t}_{1} *(\mathbb{P} y)$ and $\mathbf{t}_{2} *(\mathbb{P} y)$ becomes not legitimate (respe. becomes legitimate) then $\mathbf{t}_{1,2} *(\mathbb{P} y)$ does not make any change, where $\mathbf{t}_{1,2}$ is obtained from $\mathbf{t}$ by changing both instances of $\diamond$ (at places $n_{1}$ and $n_{2}$ ) into $\mathbb{P}$.

This gives us the general form of our additional axioms. However, as we said, to express the axioms we need nominals in the language. The challenge is to express the axioms in KR without the use of nominals, by using some other technical device. This is indeed what we are going to do now! 
2.2 Manipulating finite bi-modal Kripke models

This subsection prepares the ground for our KR completeness proof. Our strategy is to construct first a finite bi-modal tree Kripke model for the modalities $\diamond$ and $\mathbb{P}$ and then convert this model to a finite $\mathbf{K R}$ model. To achieve this, we need to learn how to manipulate finite bi-modal Kripke models. This is the task of the current subsection.

\section{Definition 2.4}

1. Let $\diamond$ and $\mathbb{P}$ be two modalities. A fintie Kripke model for these modalities has the form $\mathbf{m}=\left(S, \rho_{1}, \rho_{2}, a, h\right)$, where $S$ is the finite set of possible worlds, $a \in S$ is the initial root (actual) world and $\rho_{1}$ and $\rho_{2}$ are binary relations on $S$. We require that $\rho_{1} \cap \rho_{2}=\varnothing . h$ is an assignment giving each atomic $q$ of the language a subset $h(q) \subseteq S$.

2. $\mathbf{m}$ is said to be a tree model if $(S, \rho, a)$ is a tree with root $a$, where $\rho=\rho_{1} \cup \rho_{2}$. This means that $\rho$ is irreflexive and every node $x \in S$ has a unique path (leading up to it) of the form $\left(a, x_{1}, \ldots, x_{n}\right)$ such that $a \rho x_{1} \wedge x_{1} \rho x_{2} \wedge \ldots \wedge x_{n-1} \rho x_{n}$ holds and $x_{n}=x$.

Since $\rho=\rho_{1} \cup \rho_{2}$ we denote paths also by

$$
a \stackrel{M_{1}}{\longrightarrow} x_{1} \stackrel{M_{2}}{\longrightarrow} x_{2} \longrightarrow \ldots \stackrel{M_{n}}{\longrightarrow} x_{n}
$$

where $m_{i} \in\{\diamond, \mathbb{P}\}$ and $x \stackrel{M}{\longrightarrow} y$ means

$$
\begin{aligned}
& x \rho_{1} y \text { if } M=\diamond \text { and } \\
& x \rho_{2} y \text { if } M=\mathbb{P} .
\end{aligned}
$$

We call the above annotated paths.

We also write

$$
\mathbf{t}=\left(a, M_{1} x_{1}, \ldots, M_{n} x_{n}\right)
$$

for annotated paths.

3. Let $\mathbf{m}$ be a tree model. We define the notion of a level of a node $x \in S$ as follows: Let $n$ be the length of the maximal path in $(S, \rho, a)$, i.e., there exists $\left(a, x_{1}, \ldots, x_{n-1}\right)$ such that $a \rho x_{1} \wedge \ldots \wedge x_{n-2} \rho x_{n-1}$ and for every path $\left(a, y_{1}, \ldots, y_{m-1}\right)$ in $(S, \rho, a)$ we have $m \leq n$.

Then we define

(a) The pont $a$ is of level $n$.

(b) If $x$ is of level $n-k$ and $x \rho y$ then $y$ is of level $n-k-1$.

(c) The level of the model $\mathbf{m}$ is the level of $a$.

Note that the level of a node $x$ is $n-k$ where $k$ is its "distance" from the root $a$.

Definition 2.5 (Path nominals for bi-modal models) Let $\mathbf{m}=\left(S, \rho_{1}, \rho_{2}, a, h\right)$ be a finite tree bi-modal Kripke model. Let $\delta$ be a function associating with each $x \in S$, a formula $\delta(x)$ of the bi-modal langauge with $\diamond, \mathbb{P}$. We say $\delta$ is a system of path nominals for $\mathbf{m}$ iff the following holds:

1. $x \vDash \delta(x)$ in $\mathbf{m}$. 
2. Let $x \rho_{1} y$ and $x \rho_{1} z$ and $y \neq z$ hold then $\delta(y) \wedge \delta(z)$ has no finite tree model (i.e., they are semantically consistent).

3. If $x$ is of level $k$ then $\delta(x)$ has at most $n-k$ nested modalities.

Remark 2.6

1. Note that if a finite model $\mathbf{m}$ has a system of path nominals as in Definition 2.5, then the following holds for any $t \in S$

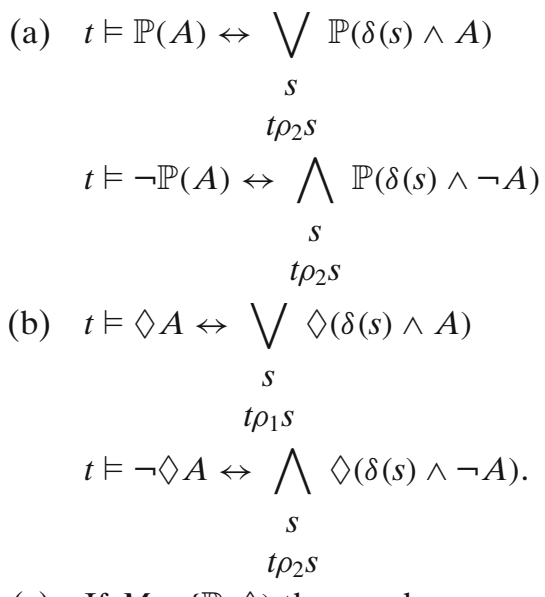

(c) If $M \in\{\mathbb{P}, \diamond)$ then we have

$$
t \vDash M(\delta(z) \wedge A) \wedge M(\delta(z) \wedge B) \leftrightarrow M(\delta(z) \wedge A \wedge B)
$$

2. Note also that the restriction (3) on $\delta$ of Definition 2.5 is not really restrictive because we have the proposition $(*)$ :

(*) If $t$ is of level $k$ then for any wff $A$ there exists a formula $A^{\prime}$ with at most $n-k$ nested modalities such that $t \vDash A \leftrightarrow A^{\prime}$.

This can be proved by induction on $n-k$, where $n$ is the level of the model $\mathbf{m}$.

Remark 2.7 Note that the concept of path nominals is intended to identify paths and not necessarily identify worlds. Consider Fig. 20, describing a model with $q=\top$ everywhere and one modality $\diamond$. We can use the system of path nominals $\delta$ as follows:

We see that $\delta(b)=\delta(d)$, so these are not the traditional nominals. However $\delta(b) \wedge$ $\delta(c)$ is not consistent. So the paths

$$
\begin{aligned}
& a \rightarrow c \rightarrow d \\
& a \rightarrow e \\
& a \rightarrow b
\end{aligned}
$$




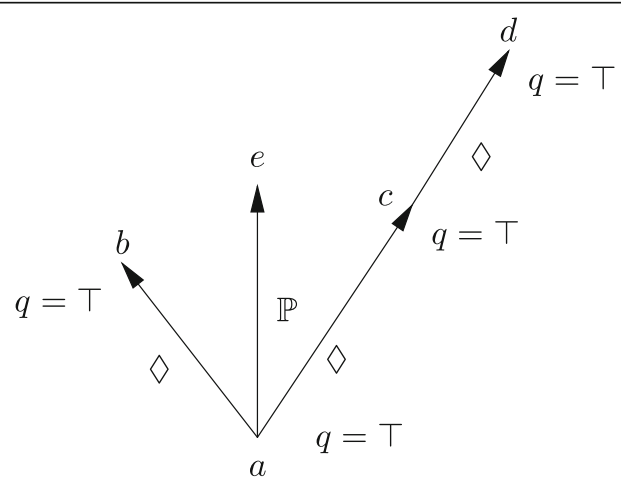

Fig. 20 Illustrating path nominals

can be identified uniquely.

$$
\begin{aligned}
& \delta(e)=\neg \diamond \top \\
& \delta(b)=\neg \diamond \top \\
& \delta(d)=\neg \diamond \top \\
& \delta(c)=\diamond \neg \diamond \top \\
& \delta(a)=\diamond \neg \diamond \top \wedge \diamond \diamond \neg \diamond \top
\end{aligned}
$$

Definition 2.8 Let $\mathbf{m}=\left(S, \rho_{1}, \rho_{2}, a, h\right)$ be a finite tree model and let $\delta$ be a system of path nominals for $\mathbf{m}$. Let $M_{i} \in\{\oslash, \mathbb{P}\}$ and let $\left(a, x_{1}, \ldots, x_{n}\right)$ be a path.

1. Let $\mathbf{t}=\left(a, M_{1} x_{1}, \ldots, M_{n} x_{n}\right)$ be an annotated path in $\mathbf{m}$. The notation means that we have $a \rho_{M_{1}} x_{1} \wedge \ldots \wedge x_{n-1} \rho_{M_{n}} x_{n}$ where

$$
\rho_{M}=\rho_{1} \text { if } M=\diamond
$$

and

$$
\rho_{M}=\rho_{2} \text { if } M=\mathbb{P} .
$$

Thus the annotated path $\mathbf{t}$ is more specific about how we get to $x_{n}$.

2. Consider the wff $\Psi_{\mathbf{t}}^{\delta}(X)$ defined as

$$
\Psi_{\mathbf{t}}^{\delta}(X)=M_{1}\left(\delta\left(x_{1}\right) \wedge M_{2}\left(\delta\left(x_{2}\right) \wedge \ldots \wedge M_{n}\left(\delta\left(x_{n}\right) \wedge X\right) \ldots\right) .\right.
$$

3. Define the modality $M^{\prime}$ as follows:

$$
\begin{aligned}
& M^{\prime}=\diamond \text { if } M=\mathbb{P} \\
& M^{\prime}=\mathbb{P} \text { if } M=\diamond .
\end{aligned}
$$

4. Let $\mathbf{t}=\left(a, M_{1}, x_{1}, \ldots, M_{n} x_{n}\right)$. Define $\mathbf{t}_{[j]}^{\prime} 1<j \leq n$ to be

$$
\mathbf{t}_{[j]}^{\prime}=\left(a, M_{1} x_{1}, \ldots, M_{j-1} x_{j-1}, M_{j}^{\prime} x_{j}, \ldots, M_{n} x_{n}\right)
$$

Given a $\mathbf{t}$ consider $\Psi_{t_{[j]}^{\prime}}^{\delta}(X)$. We call this formula a one point change of $\Psi_{\mathbf{t}}^{\delta}(X)$.

5. Let $\mathbf{t}=\left(a, M_{1} x_{1}, \ldots, M_{n} x_{n}\right)$. Assume $x_{n} \rho_{1} y$. Then the formula $\Psi_{\mathbf{t}}^{\delta}(\mathbb{P} \delta(y))$ is called a $\mathbb{P}$-test at the point $x_{n}$. 


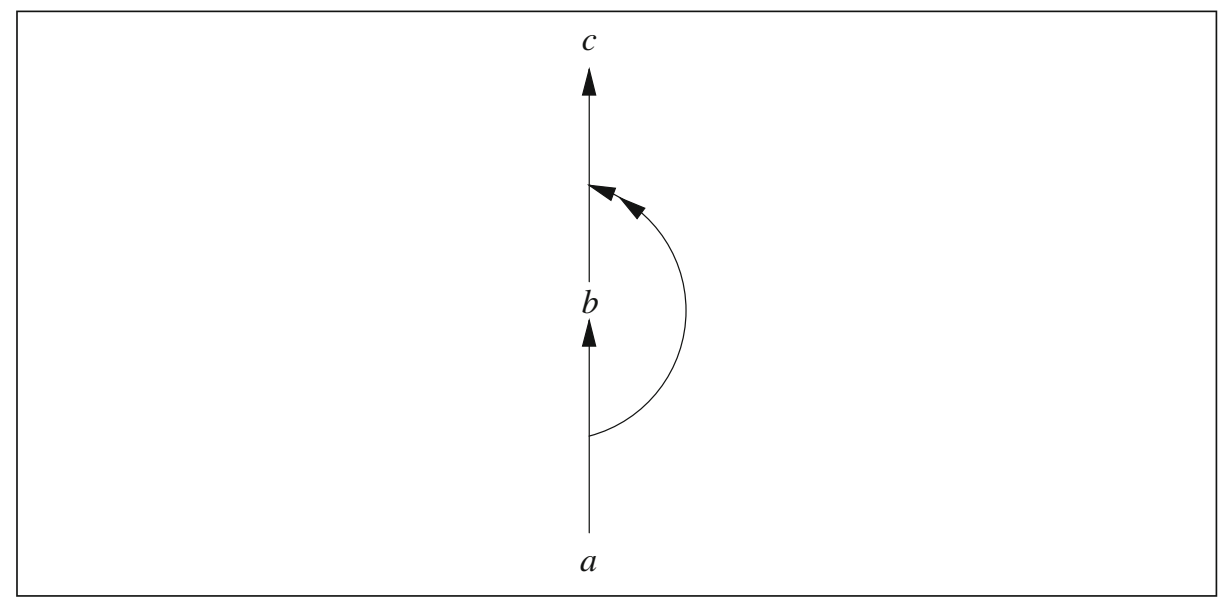

Fig. 21 Illustrating Definition 2.8

Remark 2.9 To explain the concepts of Definition 2.8, we consider the following two figures, Figs. 21 and 22.

The first figure describes a KR model for $\diamond$ and $\mathbb{P}$. The second figure describes the unfolding of this KR model into a bi-modal tree model for the two modalities $\diamond$ and $\mathbb{P}$.

A path nominal system can be defined on the model of Fig. 22 as follows:

$$
\begin{aligned}
& \delta\left(c_{1}\right)=\delta\left(c_{2}\right)=\delta\left(c_{3}\right)=\neg \diamond \top \wedge \neg \mathbb{P} \top \\
& \delta\left(b_{1}\right)=\diamond \delta\left(c_{1}\right) \wedge \mathbb{P}\left(\delta\left(c_{2}\right)\right) \\
& \delta\left(b_{2}\right)=\diamond \delta\left(c_{3}\right) \\
& \delta(a)=\diamond \delta\left(b_{1}\right) \wedge \mathbb{P} \delta\left(b_{2}\right) .
\end{aligned}
$$

If we are given the model of Fig. 22 together with $\delta$, how can we retrieve the model of Fig. 22 from the model of Fig. 21?

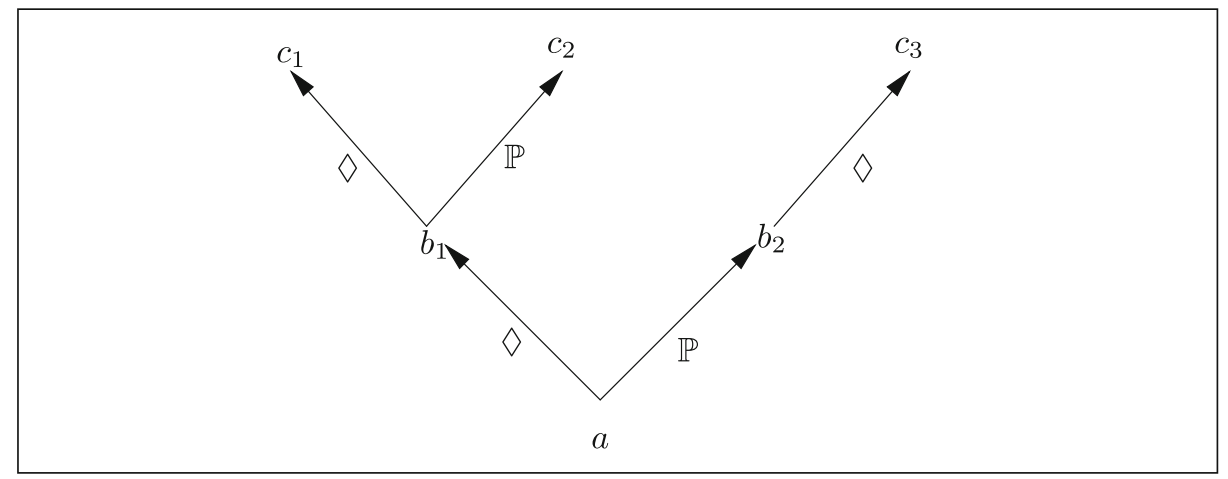

Fig. 22 Expanding Fig. 21 
As part of the conversion/retrieval process, we need to identify the effects of the double arrow

$$
(a \rightarrow b) \rightarrow(b \rightarrow c)
$$

which resides in Fig. 21 and has manifestations in Fig. 22, namely that $b_{2} \vDash \neg \mathbb{P} \delta\left(c_{3}\right)$. To achieve such an identification we use the $\mathbb{P}$-test formulas.

We formally want to convert the model of Fig. 22 into a reactive bi-modal model of Fig. 23 by adding a set $R_{2}$ of double arrows and an activity function $\mathbf{f}$. We let $R_{1}=\rho_{\diamond} \cup \rho_{\mathbb{P}}$ and let the $\mathbb{P}$ and $\diamond$ modalities traverse all connections of $R_{1}$.

Obviously, adjustments are needed!

The end result we hope is a model equivalent to that of Fig. 21.

1. Consider the formula

$$
\Psi=\neg \mathbb{P}\left(\mathbb{P} \delta\left(c_{3}\right) \wedge \delta\left(b_{2}\right)\right)
$$

and consider $\Psi^{\prime}$

$$
\Psi^{\prime}=\diamond \mathbb{P}\left(\delta\left(c_{3}\right) \wedge \delta\left(b_{2}\right)\right)
$$

We have $a \vDash \Psi$ and $a \vDash \Psi^{\prime}$, namely the value changed when we changed $\mathbb{P}$ to $\diamond$ ! This invites $\left(a \rightarrow b_{2}\right) \rightarrow\left(b_{2} \rightarrow c_{3}\right) \in R_{2}$.

2. Similarly, consider

$$
\begin{aligned}
& \left.\varphi=\diamond\left(\delta\left(b_{1}\right) \wedge \mathbb{P} \delta(c)_{2}\right)\right) \\
& \varphi^{\prime}=\neg \mathbb{P}\left(\delta\left(b_{1}\right) \wedge \mathbb{P} \delta\left(c_{2}\right)\right)
\end{aligned}
$$

We have $a \vDash \varphi$ and $a \vDash \varphi^{\prime}$.

The correct way of dealing with this is to declare $a \rightarrow b_{1}$ as off. $\diamond$ will ignore it and $\mathbb{P}$ will not be able to traverse it.

We can also in this particular case add the double arrows

$$
\left(a \rightarrow b_{1}\right) \rightarrow\left(b_{1} \rightarrow c_{2}\right)
$$

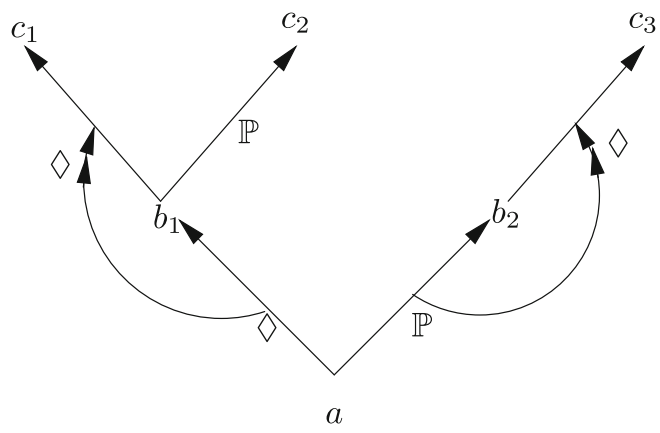

Fig. 23 Converting Fig. 22 
as well as

$$
\left(a \rightarrow b_{1}\right) \rightarrow\left(b_{1} \rightarrow c_{1}\right)
$$

3. Also consider

$$
b_{2} \vDash \diamond \delta\left(c_{3}\right)
$$

but

$$
b_{2} \vDash \neg \mathbb{P} \delta\left(c_{3}\right)
$$

This is already taken care of by (1) above. Had it been the case that $b_{2}$ had no $\mathbb{P}$ predecessors, we would have declared $b_{2} \rightarrow c_{3}$ as off! But this is not needed here.

The $\mathbb{P}$ tests show that if we change $\mathbb{P}$ to $\diamond$ or $\diamond$ to $\mathbb{P}$ the truth values switch. This calls for double arrows as discussed in Fig. 23.

Now consider the model in Fig. 23 as a KR model with the relation $R_{1}=\rho_{\mathbb{P}} \cup \rho_{\diamond}$ and $R_{2}$ as drawn. The assignment is the same as in Fig. 21, i.e., points $c_{1}, c_{2}, c_{3}$ of Fig. 23 get the same assignment as point $c$ of Fig. 21 and similarly points $b_{1}, b_{2}$ get the same as $b$, respectively.

So, when we evaluate $a \vDash \mathbb{P} A$ in Fig. 21, we go to point $b$ and activate the double arrow. When we evaluate $a \vDash \mathbb{P} A$ in Fig. 23, we either go to $b_{1}$ or go to $b_{2}$ and activate the respective double arrows in each case.

When we go to $b_{2}$, the double arrow deactivates $b_{2} \rightarrow c_{3}$ and so $\neg \mathbb{P} \delta\left(c_{3}\right)$ holds but $\diamond \delta\left(c_{3}\right)$ still holds because $\diamond$ ignores the effects of double arrows.

Similarly at $b_{1}$ we get the desired results. Thus the models of Figs. 21 and 23 are equivalent.

Remark 2.10 Note in Definition 2.8 when we define $\Psi_{\mathbf{t}}^{\delta}(X)$ for a path

$$
\mathbf{t}=\left(a, M_{1} x_{1}, \ldots, M_{n} x_{n}\right),
$$

the formula $\Psi_{\mathbf{t}}^{\delta}(X)$ does not mention the path $\mathbf{t}$. This will be significant later on, when we define double arrows using the $\Psi_{\mathbf{t}}^{\delta}$ formulas.

To understand what is happening, consider Fig. 20.

Let

$$
\begin{aligned}
& t_{1}=(a, \mathbb{P} e) \\
& t_{2}=(a, \diamond b)
\end{aligned}
$$

we have $\Psi_{t_{1}}^{\delta}=\Psi_{t_{2}}^{\delta}$.

Furthermore, if we consider

$$
t_{3}=(a, \diamond c)
$$

we have

$$
a \vDash \Psi_{t_{3}}^{\delta}(\diamond \top)
$$

but also

$$
a \vDash \Psi_{t_{1}}^{\delta}(\neg \diamond \top)
$$




\section{Definition 2.11}

1. Let $\mathbf{m}=\left(S, \rho_{1}, \rho_{2}, a, h\right)$ be a tree model. Define a relation $\equiv$ on $S$ by letting

- $\quad x \equiv y$ iff $\exists z\left(z \rho_{i} x \wedge z \rho_{i} y\right)$ and $i \in\{1,2\}$ and if $k$ is the level of $x$ and $y$ then for all $A$ of less than $n-k$ nested modalities we have $x \vDash A$ iff $y \vDash A$.

2. let $\mathbf{m}^{\prime}=\left(S^{\prime} \subseteq S, \rho_{2}^{\prime}, \rho_{2}^{\prime}, a, h\right)$ be defined as follows (we call $\mathbf{m}^{\prime}$ the "reduced version" of $\mathbf{m})$ :

(a) Let $a \in S^{\prime}$.

(b) Assume $x$ is in $S^{\prime}$, let $S_{x}^{i}$ be a choice of set of points from $S$ such that

- $x \rho_{i} z$ for $z \in S_{x}^{i}$

- If $z, z^{\prime} \in S_{x}^{i}$ and $z \neq z^{\prime}$ then $z \not \equiv z^{\prime}$.

- If $x \rho_{i} z$ then for some $z^{\prime} \in S_{x}^{i}$ we have $z \equiv z^{\prime}$.

Then let also $S_{x}^{1} \cup S_{x}^{2} \subseteq S^{\prime}$.

Let $S^{\prime}$ be the smallest subset of $S$ closed under (a) and (b). Let $\rho_{i}^{\prime}=\rho_{i} \uparrow S^{\prime}$.

3. A tree model $\mathbf{m}$ is said to be reduced if $\mathbf{m}=\mathbf{m}^{\prime}$.

4. Note that under 2(b) above, we may choose different points, but all possible models are isomorphic. This is why we speak of "the reuduced model".

Lemma 2.12 Let $\mathbf{m}=\left(S, \rho_{1}, \rho_{2}, a, h\right)$ be a tree model and let $\mathbf{m}^{\prime}=\left(S^{\prime}, \rho_{1}^{\prime}, \rho_{2}^{\prime}, a, h\right)$ be its reduced version. Assume $a$ is of level $n$. Then for any $x \in S^{\prime}$ of level $k$ and any $A$ with at most $n-k$ nested modalities we have $x \vDash A$ in $\mathbf{m}$ iff $a \vDash A$ in $\mathbf{m}$ '.

Proof The proof is by induction on $n-k$ and presents no difficulties.

Lemma 2.13 Let $\mathbf{m}$ be a reduced tree model. Assume our language is based on a finite set $\mathbb{Q}$ of atoms. Define $\delta(x), x \in S$ by $\delta(x)=\{A \mid x \vDash A$ and $A$ is based on $\mathbb{Q}$ and contains at most $n-k$ nested modalities, where $k$ is the level of $x\}$.

Then $\delta(x)$ is a system of path nominals.

Proof This follows from the facts that the model is reduced and $\delta(x)$ is a complete theory for the language of up to $n-k$ level formulas.

Lemma 2.14 Let $\mathbf{m}=\left(S, \rho_{1}, \rho_{2}, a, h\right)$ be a reduced tree model for a finite language based on $\mathbb{Q}$, and let $\delta(x)$ be the path nominal system for $\mathbf{m}$ as defined in Lemma 2.13. Let $t \in S$. Then

$$
\delta(t)=\varepsilon_{t} \wedge \bigwedge_{\pi(t)} \Psi_{\pi(t)}
$$

where $\varepsilon_{t}$ is a conjuctive form, i.e., $\varepsilon=\bigwedge_{q \in \mathbb{Q}} \pm q$ and where $\pi(t)$ ranges over all maximal paths of the form $\left(t, M_{1} x_{1}, \ldots, M_{n} x_{n}\right)$ and $\Psi_{\pi}$ is $M_{1}\left(\delta\left(x_{1}\right) \wedge M_{2}\left(\delta\left(x_{3}\right) \wedge\right.\right.$ $\left.\ldots \wedge M_{n} \delta\left(x_{n}\right) \ldots\right)$.

Note that $\Psi_{\pi}$ is $\Psi_{\pi}^{\delta}(\top)$ as defined in Definition 2.8 .

Proof By induction on $n-k$, where $k$ is the level of $t$

1. The case of $n-k=0$ is clear. 
2. The case of $n-k=1$ is also clear since any theory of a node $x$ of $n-1$ has the form $\delta(x)=\varepsilon \wedge \bigwedge_{i} \pm \mathbb{P} \varepsilon_{i} \wedge \bigwedge_{j} \pm \vee_{j}$.

3. Let $t$ be such that $x_{1}, \ldots, x_{m}$ are its $\rho_{1}$ successors and $y_{1}, \ldots, y_{r}$ are its $\rho_{2}$ successors. We have $t \rho_{1} x_{1}, \ldots, t \rho_{2} x_{m}$, and $t \rho_{2} y_{1}, \ldots, t \rho_{2} y_{r}$.

First we observe that the theory of node $t$ is equivalent to

$$
A_{t}=\varepsilon_{t} \wedge \bigwedge_{i} \diamond \delta\left(x_{i}\right) \wedge \bigwedge_{j} \mathbb{P} \delta\left(y_{j}\right)
$$

Next observe that by the induction hypothesis each $\delta(z), z \in\left\{x_{i}, y_{j}\right\}$ has the form

$$
\begin{aligned}
\delta(z) & =\varepsilon_{z} \wedge \wedge_{\eta(z)} \Psi_{\eta(z)} \\
& =\delta(z) \wedge \bigwedge_{\eta(z)} \Psi_{\eta(z)}
\end{aligned}
$$

where $\eta(z)$ ranges over all maximal paths starting at $z$.

Therefore $A_{t}$ has the form

$$
A_{t}=\varepsilon_{t} \wedge \bigwedge_{\substack{z \\ t \rho_{1} z}}\left[\diamond\left(\delta(z) \wedge \bigwedge_{\eta(z)} \psi_{\eta(z)}\right)\right] \wedge \bigwedge_{\substack{z \\ t \rho_{2} z}}\left[\mathbb{P}\left(\delta(z) \wedge \bigwedge_{\eta(z)} \psi_{\eta(z)}\right)\right]
$$

We want to rewrite form (8) of Lemma 2.14 in the form (*1) above.

To achieve that let us first write each $\pi=\left(t, M_{1} z, M_{2} z_{2}, \ldots\right)$ as $\pi=\left(t, M_{1} z, \eta\right)$.

Hence

$$
\Psi_{\pi}=M_{1}\left(\delta(z) \wedge \Psi_{\eta}\right)
$$

Second, let us use $(\sharp)$ and rewrite $(*)$ into two conjuncts:

$$
(*)=\varepsilon_{t} \wedge \bigwedge_{\pi(t)} \Psi_{\pi(t)}=\varepsilon_{t} \wedge \bigwedge_{\substack{z, \eta(z) \\ t \rho_{1} z}} \nabla\left(\delta(z) \wedge \Psi_{\eta(z)}\right) \wedge \bigwedge_{\substack{z, \eta(z) \\ t \rho_{2}}} \mathbb{P}\left(\delta(z) \wedge \Psi_{\eta(z)}\right)
$$

Now since $\delta$ is a system of path nominals (see item 1(c) of Remark 2.6): we can continue and rewrite

$$
=\varepsilon_{t} \wedge \bigwedge_{\substack{z \\ t \rho_{1} z}} \nabla\left(\delta(z) \wedge \bigwedge_{\eta(z)} \Psi_{\eta(z)}\right) \wedge \bigwedge_{\substack{z \\ t \rho_{2} z}} \mathbb{P}\left(\delta(z) \wedge \bigwedge_{\eta(z)} \Psi_{\eta(z)}\right)=(* 1)
$$

Remark 2.15 Note that the representation of the path nominals in Lemma 2.14 uses formulas of the positive (in $\mathbb{P}, \diamond$ ) form

$$
\Psi_{\pi}^{\delta}=M_{1}\left(\delta\left(x_{1}\right) \wedge\left(\cdots M_{n} \delta\left(x_{n}\right)\right) \ldots\right) .
$$

We do not use $\neg M$ in $\Psi_{\pi}$. So we do not use formulas of the form

$$
M_{1}\left(\delta ( x _ { 1 } ) \wedge \left(\ldots\left(M_{n}\left(\delta\left(x_{n}\right) \wedge \neg M \delta(y)\right) \ldots\right)\right.\right.
$$

We now want to give definitions and theorems which convert a finite bi-modal model $\mathbf{m}$ into an equivalent finite reactive model $\mathbf{m}^{\sharp}$ (as in Definition 2.1). We need 
to develop some definitions of path nominals for such models. So we will define the notions of legitimate paths and path nominals for the models of Definition 2.1.

Definition 2.16 (Legitimate paths) Let $\mathbf{m}=\left(S, R_{1}, R_{2}, \mathbf{f}, a, h\right)$ be a $\mathbf{K R}$ model as in Definition 2.1. We define by induction the notion of a legitimate path $\mathbf{t}=$ $\left(a, M_{1} x_{1}, \ldots, M_{n} x_{n}\right)$, where $a R_{1} x_{1} \wedge \ldots x_{n-1} R_{1} x_{n}$ hold and $M_{i} \in\{\diamond, \mathbb{P}\}, i=1, \ldots, n$. We also define the functions $\mathbf{f}_{t}, \mathbf{t}$ a legitimate path.

1. (a) is a legitimate path and $\mathbf{f}_{(a)}=\mathbf{f}$.

2. Assume that $\mathbf{t}=\left(a, M_{1} x_{1}, \ldots, M_{n} x_{n}\right)$ is a legitimate path and assume that $\mathbf{f}_{\left(a, x_{1}, \ldots, x_{n}\right)}$ has been defined. Let $y$ be such that $x_{n} R_{1} y$ holds. Then $\left(a, M_{1} x_{1}, \ldots, M_{n} x_{n}, \diamond y\right)$ is a legitimate path and let $\mathbf{f}_{\mathbf{t} *(\diamond y)}=\mathbf{f}_{\mathbf{t}}$.

3. Assume as in (2) that $\mathbf{t}=\left(a, M_{1} x_{1}, \ldots, M_{n} x_{n}\right)$ is a legitimate path and that $\mathbf{f}_{\mathbf{t}}$ has been defined. Let $y$ be such that $x_{n} R_{1} y$ and that $\mathbf{f}_{\mathbf{t}}\left(x_{n}, y\right)=1$. Then $\mathbf{s}=\left(a, M_{1} x_{1}, \ldots, M_{n} x_{n}, \mathbb{P} y\right)$ is a legitimate path, and let $\mathbf{f}_{\mathbf{s}}=\mathbf{f}_{\mathbf{t}}\left[x_{m}, y\right]$.

4. A legitimate path is maximal for $M$ if it has the form $\left(a, M_{1} x_{1}, \ldots, M_{n} x_{n}, \neg M y\right)$. This means that $x_{n}$ is an endpoint, as far as $M$ is concerned.

5. Let $S^{\pi}$ be the set of all legitimate paths of $S$.

Definition 2.17 (Path nominals for reactive models) Let $\mathbf{m}=\left(S, R_{1}, R_{2}, \mathbf{f}, a, h\right)$ be a finite KR model. Let $\delta$ be a function defined on $S^{\pi}$ giving for each legitimate path $\mathbf{t}=\left(a, M_{1} x_{1}, \ldots, M_{n} x_{n}\right)$ a wff $\delta_{\mathbf{t}}$ of the logic such that the following hold:

1. Let $y, z$ be such that $x_{n} R_{1} y \wedge x_{n} R_{1} z \wedge y \neq z$. Let $\mathbf{s}_{1}=\mathbf{t} *(M y)$ and $s_{2}=\mathbf{t} *$ $(M z), M \in\{\oslash, \mathbb{P}\}$. Then $\delta_{\mathbf{s}_{1}} \wedge \delta_{\mathbf{s}_{2}}$ has no reactive model, i.e., it is semantically not consistent.

2. $\mathbf{t} \vDash \delta_{\mathbf{t}}$ in the model $\mathbf{m}_{\mathbf{t}}=\left(S, R_{2}, R_{2}, \mathbf{f}_{\mathbf{t}}, t, h\right)$.

3. Note that from (1) and (2) above it follows that for $\mathbf{t}_{i}=\left(a, M_{1} x_{1}, \ldots, M_{i} x_{i}\right), i=$ $1, \ldots, n$ we have

$$
\begin{aligned}
& a \vDash M_{1}\left(\delta _ { \mathbf { t } _ { 1 } } \wedge M _ { 2 } \left(\delta _ { \mathbf { t } _ { 2 } } \wedge \left(\ldots \wedge M_{(n-1)}\left(\delta_{\mathbf{t}_{n-1}} \wedge M_{n}\left(\delta_{\mathbf{t}_{n}} \wedge A\right) \ldots\right)\right.\right.\right. \\
& \text { in } \mathbf{m}=\left(S, R_{1}, R_{2}, \mathbf{f}, a, h\right) \text { iff } \mathbf{t} \vDash A \text { in } \mathbf{m}_{\mathbf{t}}=\left(S, R_{1}, R_{2}, \mathbf{f}_{\mathbf{t}}, \mathbf{t}, h\right) .
\end{aligned}
$$

In other words, $\delta_{\mathbf{t}_{i}}$ act as nominals for the path $\mathbf{t}$.

4. It will be convenient for us in the future to use another function $\delta_{t}^{*}$ associating with each $\mathbf{t}$ a distict atomic formula $q_{\mathbf{t}}$. We can abuse notation and write ' $\delta_{\mathbf{t}}^{*}$ ' for ' $q_{\mathbf{t}}$ '.

The atoms $q_{\mathbf{t}}$ (or $\delta_{\mathbf{t}}^{*}$ if we abuse notation) are intended to act as path nominals.

Definition 2.18 Let $\mathbf{m}=\left(S, \rho_{2}, \rho_{2}, a, h\right)$ be a reduced finite tree model based on a finite set of atoms. By Lemmas 2.13 and 2.14

$$
\delta(t)=\varepsilon_{t} \wedge \bigwedge_{\pi(t)} \Psi_{\pi(t)}
$$

is a path nominal system for $\mathbf{m}$. Let $R_{1}=\rho_{1} \cup \rho_{2}$. We shall now define a set $R_{2}$ of double arrows for $\mathbf{m}$, as well as an activity function $\mathbf{f}$. 
Let $t \in S$ and let

$$
\begin{aligned}
& \pi=\left(t, \mathbb{P} x_{1}, M_{2} x_{2}, \ldots, M_{r} x_{r}\right) \\
& \pi^{\prime}=\left(t, \diamond x_{1}^{\prime}, M_{2} x_{2}^{\prime}, \ldots, M_{r} x_{r}^{\prime}\right)
\end{aligned}
$$

for $r \geq 1$.

Assume for $i=1, \ldots, r$

$$
\delta\left(x_{i}\right)=\delta\left(x_{i}^{\prime}\right)
$$

We distinguish several cases.

(a) Case 1. For some $y$ such that $x_{r} \rho_{2} y$ (and therefore $t \vDash \Psi_{\pi}^{\delta}(\mathbb{P} \delta(y))$ ) we have $t \vDash$ $\Psi_{\pi^{\prime}}^{\delta}(\neg \mathbb{P} \delta(y))$.

In this case introduce the double arrow $\left(t \rightarrow x_{1}\right) \rightarrow\left(x_{r} \rightarrow y\right)$ into $R_{2}$.

(b) Case 2. $u \rho_{1} v$ holds.

In this case let $\mathbf{f}(u \rightarrow v)=0$.

So all $\rho_{1}$ arrows are off.

(c) Note following Case 2. Note that since Case 1 introduces double arrows only to $\rho_{2}$ connections, the function $\mathbf{f}$ does not change its 'off' value on all $\rho_{1}$ connections!

Consider now the model $\mathbf{m}^{\sharp}=\left(S, R_{1}, R_{2}, \mathbf{f}, a, h\right)$. Call this model the reactive conversion of $\mathbf{m}$. In this model we read $\mathbb{P}$ and $\diamond$ as in Definition 2.2.

Remark 2.19

1. Part 1. The question. Consider model $\mathbf{m}$ of Definition 2.18 and its reactive conversion $\mathbf{m}^{\sharp}$. Take a $t \in S$ of level $k$ and a wff $A$ of at most $n-k$ nested modalities.

Question. Do we have $(\sharp 1)$ below?

$(\sharp 1) \quad t \vDash A$ in $\mathbf{m}$ iff $t \vDash A$ in $\mathbf{m}^{\sharp}$.

To be able to prove that we need some properties (axioms) of $\diamond, \mathbb{P}$ which will manifest themselves as properties of $R_{2}$. With the right axioms ( $\left.\sharp 1\right)$ might hold. Let us examine $(\sharp 1)$ more closely. It is clear immediately that $(\sharp 1)$ is not properly formualted. The model $\mathbf{m}^{\sharp}$ is a reactive model and so we cannot ask for $t \in S$ the simple question, does $t \vDash A$ in $\mathbf{m}^{\sharp}$, we need to know the legitimate path leading to $t$. So let

$$
\mathbf{t}=\left(a, M_{1} x_{1}, \ldots, M_{r} x_{r}\right)
$$

where $x_{r}=t$. We ask does $(\sharp 2)$ hold?

$(\sharp 2) \quad t \vDash A$ in $\mathbf{m}$ iff $\mathbf{t} \vDash A$ in $\mathbf{m}^{\sharp}$.

Note that the question is not simple. Consider Fig. 24 describing a model $\mathbf{m}$ (we are not showing $h$ ):

The model $\mathbf{m}$ has two maximal paths

$$
a \stackrel{\mathbb{P}}{\longrightarrow} b \stackrel{\mathbb{P}}{\longrightarrow} c
$$

and

$$
a \stackrel{\diamond}{\longrightarrow} d .
$$




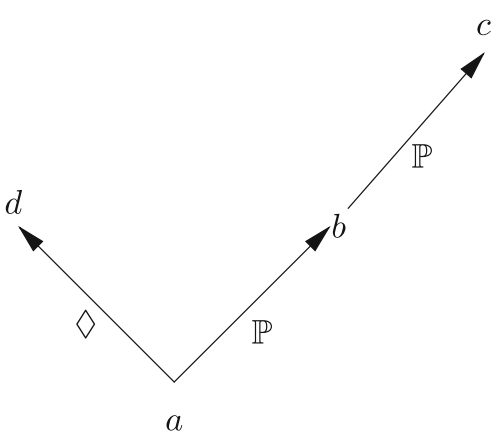

Fig. 24 Sample model

When we convert it to the model $\mathbf{m}^{\sharp}$ (we need to know the assignment $h$ to convert and define $R_{2}$ and $\mathbf{f}$, but let us ignore that) we may get new paths, such as

$$
\begin{aligned}
& a \stackrel{\diamond}{\longrightarrow} b \stackrel{\mathbb{P}}{\longrightarrow} c \\
& a \stackrel{\diamond}{\longrightarrow} b \stackrel{\diamond}{\longrightarrow} c \\
& a \stackrel{\mathbb{P}}{\longrightarrow} b \stackrel{\diamond}{\longrightarrow} c
\end{aligned}
$$

and

$$
a \stackrel{\mathbb{P}}{\longrightarrow} d
$$

An examination of Case 2 of Definition 2.18 indicates that $a \stackrel{\mathbb{P}}{\longrightarrow} d$ is not allowed but the other paths are allowed.

2. Part 2. The answer. What we need to show then are two items

item (a) The double arrows in $\mathbf{m}^{\sharp}$ behave correctly.

item (b) The new paths obtained by allowing $\diamond$ to traverse $\mathbb{P}$ connections for example, from $t$ to $s$, (i.e., $t \stackrel{\mathbb{P}}{\longrightarrow} s$ ), do not change the value of $\delta(t)$ at points $t$. Only the old paths (those already in $\mathbf{m}$ ) do count for the value of $\delta(t)$.

Let us elaborate more on (item a) and (item b).

We start with (item a):

Let $\pi=\left(a, M_{1} x_{1}, \ldots, \mathbb{P} x_{j}, \ldots, M_{r} x_{r}\right)$ be a fixed path which has $\mathbb{P}$ at the $j$ position as indicated.

Let $\pi_{j}^{\prime}=\left(a, M_{1} x_{1}, \ldots, \diamond x_{j}, \ldots, M_{r} x_{r}\right)$.

Let $x_{r} \rho_{2} y$ hold.

Assume in the model $\mathbf{m}$ we have $a \vDash \Psi_{\pi}^{\delta}(\mathbb{P} \delta(y))$.

Let $P^{\pi}$ be the set of all $j$ in $\{1, \ldots, r\}$ such that $\pi$ has $\mathbb{P}$ in the $j$ position.

Let $I, J \subseteq P^{\pi}$ be two disjoint sets such that $I \cup J=P^{\pi}$ and

(a) $j \in J \rightarrow a \vDash \Psi_{\pi_{j}^{\prime}}^{\delta}(\neg \mathbb{P} \delta(y))$

(b) $j \in I \Rightarrow a \vDash \Psi_{\pi_{j}^{\prime}}^{\delta}(\mathbb{P} \delta(y))$.

Then the number of elements in $J$ is even. 
This calls for the following axiom:

$$
\Psi_{\pi}^{r}\left(\mathbb{P} \delta(y) \wedge \bigwedge_{j \in J} \Psi_{\pi_{j}^{\prime}}^{\delta}(\neg \mathbb{P} \delta(y)) \wedge \bigwedge_{j \in I} \Psi_{\pi_{j}^{\prime}}^{\delta}(\mathbb{P} \delta(y)) \rightarrow \perp\right.
$$

for any $\pi=\left(a, M_{1} x_{1}, \ldots, M_{r} x_{r}\right)$ and where $x_{r} \rho_{2} y$ holds and $P^{\pi}$ and $I, J$ are defined as above, and $J$ has an odd number of elements.

The reason that $J$ must be even is that if we traverse the path from $a$ to $t$ and trigger all double arrows as defined in Case 1 of Definition 2.18 then when we get to $x_{r}$ we still have $x_{r} \vDash \mathbb{P} \delta_{y}$ ) (since $x_{r} \rho_{2} y$ holds). So the double arrows must cancel each other, so $J$ must be even.

In fact, the situation is much more complex than that as we shall now analyse.

3. Part 3. The axiom. Let $t \in S$. Let $\pi=\left(a, M_{1} x_{1}, \ldots, M_{r} x_{r}\right)$ be the unique path in $\mathbf{m}$ leading up to $x_{r}$. Let $\pi^{*}=\left(a, M_{1}^{*} x_{1}, \ldots, M_{r}^{*} x_{r}\right)$ be a legitimate path in $\mathbf{m}^{\sharp}$ leading to $x_{r}$ such that $\pi^{*}$ is obtained from $\pi$ by changing some $\mathbb{P}$ into $\diamond$.

Assume that $x_{r} \stackrel{\mathbb{P}}{\longrightarrow} y$ in $\mathbf{m}$. We want to make sure that $\mathbf{f}_{\pi^{*}}\left(x_{r} \rightarrow y\right)=1$ in $\mathbf{m}^{\sharp}$. Figure 25 shows what we need to worry about

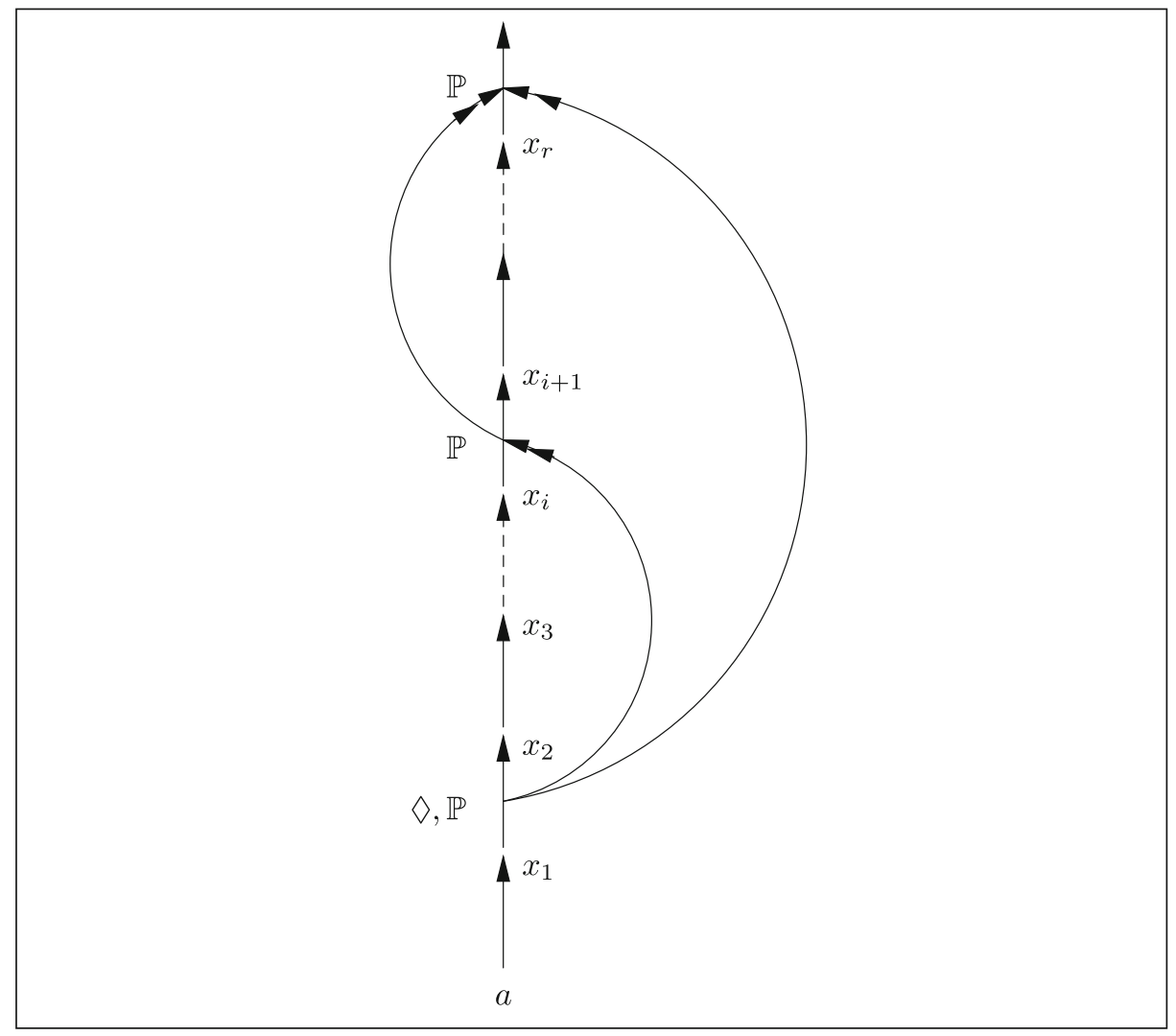

Fig. 25 Illustrating a challenge for our axioms 
The path $\pi$ in $\mathbf{m}$ contains $x_{1} \stackrel{\mathbb{P}}{\longrightarrow} x_{2}$. This connection sends a double arrow to $x_{r} \stackrel{\mathbb{P}}{\longrightarrow} y$ and also to $x_{i} \stackrel{\mathbb{P}}{\longrightarrow} x_{i+1}$. If $\pi^{*}$ goes through $x_{1} \stackrel{\mathbb{P}}{\longrightarrow} x_{2}$ in $\mathbf{m}^{\sharp}$ by using possible $\diamond$, (i.e., $\diamond x_{2} \in \pi^{*}$ ), then the double arrows

$$
\left.\left(x_{1}\right) \rightarrow x_{2}\right) \rightarrow\left(x_{i} \rightarrow x_{i+1}\right)
$$

and

$$
\left(x_{1} \rightarrow x_{2}\right) \rightarrow\left(x_{r} \rightarrow y\right)
$$

are not activated and no signal is sent to the $\operatorname{arc} x_{r} \stackrel{\mathbb{P}}{\longrightarrow} y$. So there are several possibilities of what can happen in $\mathbf{m}^{\sharp}$.

(a) $x_{i} \rightarrow x_{i+1}$ gets 'hit' by an odd number of active double arrows which were activated by $\pi^{*}$ on its way to $x_{i}$, and is not active in $\mathbf{m}^{\sharp}$ and therefore the only way that $\pi^{*}$ gets through to $x_{r}$ is because it uses $x_{i} \stackrel{\diamond}{\longrightarrow} x_{i+1}$, (i.e., $\diamond x_{i+1}$ is in $\left.\pi^{*}\right)$.

(b) $\quad x_{i} \stackrel{\mathbb{P}}{\longrightarrow} x_{i+1}$ is active in $\mathbf{m}^{\sharp}$ (receives an even number of double arrows) and $\pi$ gets through $x_{i} \rightarrow x_{i+1}$ either as $\diamond$ or as $\mathbb{P}$.

(c) Depending on how $\pi^{*}$ passes through $x_{i} \rightarrow x_{i+1}$ we will know whether the double arrow

$$
\left(x_{i} \rightarrow x_{i+1}\right) \rightarrow\left(x_{r} \rightarrow y\right)
$$

is activated in $\mathbf{m}^{\sharp}$ or not and so whether $x_{r} \stackrel{\mathbb{P}}{\longrightarrow} y$ is 'hit' by it.

We need to show that the net effects in $\mathbf{m}^{\sharp}$ of all double arrows received by $x_{r} \rightarrow y$ from any active and $\mathbb{P}$-traversed $x_{i} \rightarrow x_{i+1}$ in $\pi^{*}$ is that their number is even and therefore $x_{r} \rightarrow y$ is active in $\mathbf{m}^{*}$. We need to write an axiom to ensure that outcome.

We need some notation:

(a) Given a path $\pi=\left(a, M_{1} x_{1}, \ldots, M_{r} x_{r}, \mathbb{P} y\right)$ let

$$
\begin{gathered}
\pi(i, j)=\left(x_{i}, M_{i+1} x_{i+1}, \ldots, M_{j} x_{j}\right) \\
\Psi_{\pi(i, j)}^{\delta}\left(\mathbb{P} \delta\left(x_{j+1}\right)\right)=\left(\mathbb { P } \left(\delta ( x _ { i + 1 } ) \wedge M _ { i + 2 } \left(\delta \left(x_{i+2} \wedge \ldots M_{j}\left(\delta\left(x_{j}\right) \wedge \mathbb{P} \delta\left(x_{j+1}\right) \ldots\right)\right.\right.\right.\right. \\
\Psi_{\pi(i, j)}^{* \delta}\left(\neg \mathbb{P} \delta\left(x_{j+1}\right)\right)=\left(\diamond \left(\delta ( x _ { i + 1 } ) \wedge M _ { i + 2 } \left(\delta\left(x_{i+2}\right) \wedge \ldots\right.\right.\right. \\
\wedge M_{j}\left(\delta\left(x_{j}\right) \wedge \neg \mathbb{P} \delta\left(x_{j+1}\right) \ldots\right)
\end{gathered}
$$

Let $E^{\pi}$ be all $(i, j)$ such that $1 \leq i<k, 1<j \leq k$ and there exists a double arrow $\left(x_{i} \rightarrow x_{i+1}\right) \rightarrow\left(x_{j} \rightarrow x_{j+1}\right)$. This means that in $\mathbf{m}$ we have $x_{i} \vDash \alpha_{i, j\left(\pi, \pi^{*}\right)}$ where

$$
\alpha_{i, j\left(\pi, \pi^{*}\right)}=\Psi_{\pi(i, j)}^{\delta}\left(\mathbb{P} \delta\left(x_{j+1}\right)\right) \wedge \Psi_{\pi(i, j)}^{* \delta}\left(\neg \mathbb{P} \delta\left(x_{j+1}\right)\right)
$$

Let $\pi_{i}=\left(a, M_{1} x_{1}, \ldots, M_{i} x_{i}\right)$. Then we have $x_{i} \vDash \alpha_{i, j\left(\pi, \pi^{\prime}\right)}$ iff $a \vDash$ $\Psi_{\pi_{i}}^{\delta}\left(\alpha_{\left.i, j\left(\pi, \pi^{\prime}\right)\right)}\right.$.

Let $\pi^{*}=\left(a, M_{1}^{*} x_{1} \ldots M_{r}^{*} x_{r}, \mathbb{P} y\right)$.

Let $D^{\pi^{*} \pi}$ be the set of all $i$ such that $x_{i} \stackrel{\mathbb{P}}{\longrightarrow} x_{i+1}$ is in both $\pi$ and in $\pi^{*}$. 
Define the predicate Active $\left(x_{s} \rightarrow s_{s+1}, \pi, \pi^{*}\right)$ by

- $\operatorname{Active}\left(\left(x_{1} \rightarrow x_{2}\right), \pi, \pi^{*}\right)$

- $\operatorname{Active}\left(\left(x_{s+1} \rightarrow x_{s+2}\right), \pi, \pi^{*}\right)$ iff the set $\left\{i \mid 1 \leq i \leq s\right.$ and Active $\left(\left(x_{i} \rightarrow\right.\right.$ $\left.\left.x_{i+1}\right), \pi, \pi^{*}\right)$ and $(i, s) \in E^{\pi}$ and $\left.i \in D^{\pi, \pi^{*}}\right\}$ has an even number of elements.

To write an axiom $\mathbb{R} \mathbb{A}$ expressing what is needed ( $\mathbb{R} \mathbb{A}$ for reactive axiom) we need to translate the predicate $\operatorname{Active}\left(x_{s} \rightarrow x_{s+1}\right)$ into logic. This we do as follows: "Active" translates "Active"

- $\operatorname{Active}\left(\left(x_{1} \rightarrow x_{2}\right), \pi, \pi^{*}\right)=\top$

- $\operatorname{Active}\left(\left(x_{s+1} \rightarrow x_{s+2}\right), \pi, \pi^{*}\right)=$

$$
\bigwedge_{\substack{1 \leq i \leq s \\ i \in D^{\pi, \pi^{*}}}}\left[\operatorname{Active}\left(\left(x_{i} \rightarrow x_{i+1}\right), \pi, \pi^{*}\right) \wedge \Psi_{\pi_{i}}^{\delta}\left(\alpha_{i, s}\left(\pi, \pi^{*}\right)\right)\right] .
$$

If the set $\left\{i \mid 1 \leq i \leq s\right.$, such that $\delta(a) \vdash \operatorname{Active}\left(\left(x_{i} \rightarrow x_{i+1}\right), \pi, \pi^{*}\right)$ in $\mathbf{m}$ and $(i, s) \in E^{\pi}$ and $\left.i \in D^{\pi, \pi^{*}}\right\}$ has an even number of elements and

$$
=\operatorname{Active}\left(\left(x_{s} \rightarrow x_{s+1}\right), \pi, \pi^{*}\right)=\perp \text { otherwise. }
$$

Where in classical logic 'the set of the true propositions $\left\{a_{1}, \ldots, a_{k}\right\}$ has an even number of elements' can be written as

$$
\left.\left.\left[\left(\ldots\left(\neg a_{1} \rightarrow \neg a_{2}\right) \rightarrow \neg a_{3}\right) \rightarrow \ldots\right) \rightarrow \neg a_{n}\right)\right]
$$

The axiom $\mathbb{R A}$ is

$$
\mathbb{R} \mathbb{A}=\bigwedge_{\pi^{*}, \pi}\left[\Psi_{\pi}^{\delta}\left(\mathbb{P}(\delta(y)) \rightarrow \operatorname{Active}\left(\left(x_{r} \rightarrow y\right), \pi, \pi^{*}\right)\right]\right.
$$

Note that there is a restriction on $\pi^{*}$ that it only allowed to change some $\mathbb{P} x_{i}$ in $\pi$ into a $\diamond x_{i}$ in $\pi^{*}$.

Note that the axiom $\mathbb{R} \mathbb{A}$ was defined using satisfaction in the model $\mathbf{m}$ through its system $\delta$ of path nominals. To write a syntactic axiom for the completeness proof we need to use variables for the syntactical description of $\mathbf{m}$ and define $\delta$ syntactically. Let $\mu_{\mathbf{m}}, \delta_{\mathbf{m}}$ be the wffs doing this job. Then $\mu \rightarrow \mathbb{R A}(\mu)$ is an axiom for each $\mu$. This is done in Section 3.

This will give us an infinite set of axioms for each syntactical finite tree with two successor functions.

4. Part 4. Completeness. To explain (item b), consider Fig. 26

Figure 26 describes the situation in model $\mathbf{m}$. In this model we have $t \vDash \mathbb{P} \delta\left(s_{2}\right)$ and $t \vDash \diamond \delta\left(s_{1}\right)$. If in $\mathbf{m}^{\sharp}$ we allow $\diamond$ to traverse also $t \stackrel{\mathbb{P}}{\longrightarrow} s_{2}$, we may have a problem. Maybe we have that $t \models \neg \diamond \delta\left(s_{2}\right)$ holds in $\mathbf{m}$ but if we allow $\diamond$ to get to $s_{2}$ we will we get a contradiction? The answer is that we need not worry. In $\mathbf{m}^{\sharp}$, when we go to $s_{2}$ from $t$ we trigger some double arrows and so we can prove that in $\mathbf{m}^{\sharp} s_{2} \not \models \delta\left(s_{2}\right)$ (i.e., the model changes). We also show that if there are no double arrows emanating from $t \stackrel{\mathbb{P}}{\longrightarrow} s_{2}$, then $t \vDash \neg \diamond \delta\left(s_{2}\right)$ cannot be the case.

As for traversing $t \stackrel{\mathbb{P}}{\longrightarrow} s_{1}$ in $\mathbf{m}^{\sharp}$, this has been forbidden by Case 2 of Definition 2.18. 


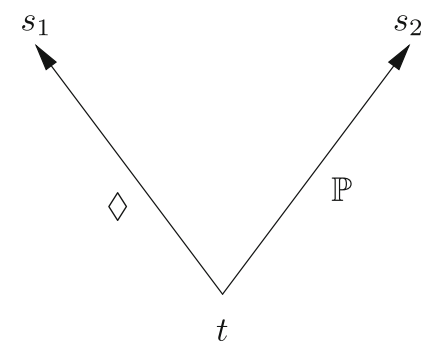

Fig. 26 Explaining our axioms

We are now ready to state and prove our main lemma.

Lemma 2.20 Let $\mathbf{m}$ and $\mathbf{m}^{\sharp}$ be two models as in Remark 2.19 and assume that $\mathbf{m}$ satisfies axiom $\mathbb{R} A$ of this remark.

Then the following condition $(\sharp)$ holds for any path $\pi=\left(a, M_{1} x_{1}, \ldots, M_{r} x_{r}\right)$ in $\mathbf{m}$ and path $\pi^{*}$ in $\mathbf{m}^{\sharp}$

$$
\pi^{*}=\left(a, M_{1}^{*} x_{1}, \ldots, M_{r}^{*} x_{r}\right)
$$

where $\pi$ and $\pi^{*}$ are related by the condition $: M_{i}^{*}=\mathbb{P} \Rightarrow M_{i}=\mathbb{P}$.

$(\sharp) \quad x_{r} \vDash \delta\left(x_{r}\right)$ in $\mathbf{m}$ iff $x_{r} \vDash \delta\left(x_{r}\right)$ in $\mathbf{m}_{\pi^{*}}^{\sharp}=\left(S, R_{1}, R_{2}, \mathbf{f}_{\pi^{*}}, a, h\right)$

where $\mathbf{f}_{\pi^{*}}=\mathbf{f}\left[a, x_{1}\right]\left[x_{1}, x_{2}\right] \ldots\left[x_{r-1}, x_{r}\right]$, as defined in Definition 2.1.

Proof By induction on the level $n-r$, where $n$ is the level of $a$.

Case $(n-r)=0$

In this case $\delta\left(x_{r}\right)$ is a classical model (i.e., $\varepsilon$ is a conjunctive normal form of atoms from $\mathbb{Q}$ ) and so $(\sharp)$ holds.

Case $(n-r)=1$

In this case $\delta\left(x_{r}\right)$ has the form

$$
\varepsilon \wedge \bigwedge_{i}\left(\diamond \varepsilon_{i} \wedge \mathbb{P} \varepsilon_{i}\right) \wedge \bigwedge_{j} \diamond\left(\eta_{j} \wedge \neg \mathbb{P} \eta_{j}\right)
$$

where $\varepsilon_{i}$ and $\eta_{j}$ are classical models.

The same holds in $\mathbf{m}^{\sharp}$ because $\mathbb{P} A \rightarrow \diamond A$ holds for $A$ not containing $\mathbb{P}$ and in $\mathbf{m}^{\sharp}$ all arrows of the form $u \stackrel{\diamond}{\longrightarrow} v$ are off.

See Case 2 of Definition 2.18 and the note there following Case 2.

Case $(n-r)>1$

The cases to check are those of $\diamond$ and $\mathbb{P}$.

Subcase of $\diamond \quad$ Let $x_{r} \rho_{1} y$ hold in $\mathbf{m}$. Then $x_{r} \vDash \diamond \delta(y)$ in $\mathbf{m}$. Hence $y \vDash \delta(y)$ in $\mathbf{m}$ and by the induction hypothesis, $y \vDash \delta(y)$ in $\mathbf{m}_{\left(\pi^{*}, \diamond y\right)}^{\sharp}$. 
Since in $\mathbf{m}^{\sharp}$ we have $x_{r} R_{1} y$, we also get that $x_{r} \vDash \diamond \delta(y)$ in $\mathbf{m}_{\pi^{*}}^{\sharp}$.

Assume now that $x_{r} \rho_{2} z$ holds in $\mathbf{m}$ and that $x_{r} \vDash \neg \diamond \delta(z)$ in $\mathbf{m}$ and of course $x_{r} \vDash$ $\mathbb{P} \delta(z)$ in $\mathbf{m}$. Look at $x_{r}$ from the point of view of $\mathbf{m}_{\pi^{*}}^{\sharp}$; we see that $x_{r} R_{1} z$ holds and therefore we have to ensure that we do not have that $\delta(z)$ holds at $z$ in $\mathbf{m}_{\left(\pi^{*}, \diamond z\right)}^{\sharp}$.

Note that the models $\mathbf{m}_{\left(\pi^{*}, \diamond z\right)}^{\sharp}$ and $\mathbf{m}_{\left(\pi^{*}, \mathbb{P} z\right)}^{\sharp}$ may not be the same because in the model $\mathbf{m}_{\left(\pi^{*}, \mathbb{P} z\right)}^{\sharp}$ the activity function is $\mathbf{f}_{\pi^{*}}\left[x_{r}, z\right]$, while in the model $\mathbf{m}_{\left(\pi^{*} \diamond z\right)}^{\sharp}$ the activity function if $\mathbf{f}_{\pi^{*}}$. We know by the induction hypothesis that in the model $\mathbf{m}_{\left(\pi^{*}, \mathbb{P} z\right)}^{\sharp}$ we do have that $z \vDash \delta(z)$.

The question is does $z \vDash \delta(z)$ hold in $\mathbf{m}_{\left(\pi^{*}, \diamond z\right)}^{\sharp}$ ? We want it not to hold.

This we now proceed to prove. We consider two possibilities for the arc $x_{r} \stackrel{\mathbb{P}}{\longrightarrow} z$.

Possiblity 1 There is at least one double arrow emanating from $x_{r} \stackrel{\mathbb{P}}{\longrightarrow} z$ into the model $\mathbf{m}_{\left(\pi^{*}, \mathbb{P} z\right)}^{\sharp}$. This means that for some $\left(M_{1} w_{1}, \ldots, M_{s} w_{s}\right)$ we have $s \rho_{1} y$ and $\Psi_{\left(x_{r}, \mathbb{P} z, M_{1} w_{1}, \ldots, M_{s} w_{s}\right)}^{\delta}(\mathbb{P} \delta(y))$ holds at $x_{r}$ while $\Psi_{\left(x_{r}, \diamond z, M_{1} w_{1}, \ldots, M_{s} w_{s}\right)}^{\delta}(\neg \mathbb{P} \delta(y))$ also holds at $x_{r}$.

We can assume that there is no double arrow of the form $\left(x_{r} \rightarrow z\right) \rightarrow\left(w_{i} \rightarrow w_{i+1}\right)$ for $i<s$. This ensures that $s$ is minimal and we don't have to worry about other double arrow changes along the way from $x_{r}$ to $s$ !

This means by Definition 2.18 that $(x \rightarrow z) \rightarrow\left(w_{s} \rightarrow y\right) \in R_{2}$.

We now consider $\delta\left(x_{r}\right)$. According to Lemma 2.14, we have that

$$
\delta\left(x_{r}\right) \vdash \diamond \Psi_{\left(M_{1} w_{1}, \ldots, M_{s} w_{s}\right)}^{\delta}(\neg \mathbb{P} \delta(y))
$$

because

$$
x_{r} \vDash \Psi_{\left(x_{r}, \diamond z, M_{1} w_{1}, \ldots, M_{s} w_{s}\right)}^{\delta}(\neg \mathbb{P} \delta(u))
$$

in m. Thus $\delta(z) \vdash \Psi_{\left(M_{1} w_{1}, \ldots, M_{s} w_{s}\right)}^{\delta}(\neg \mathbb{P} \delta(y))$.

On the other hand in the model $\mathbf{m}_{(\pi, \diamond z)}^{\sharp}$ we have that $z \vDash \Psi_{\left(M_{1} w_{1}, \ldots, M_{s} w_{s}\right)}^{\delta}(\mathbb{P} \delta(y))$ because the double arrow from $\left(x_{r} \rightarrow z\right)$ to $w_{s} \rightarrow y$ is ignored by $\diamond$.

Therefore in the model $\mathbf{m}_{\pi^{*}}^{\sharp}$ we do not have $x_{r} \vDash \diamond \delta(z)$.

Possiblity 2 No double arrows emanate from $x_{r} \rightarrow z$. In this case for all $\left(M_{1} w_{1}, \ldots, M_{s} w_{s}\right) \quad x_{r} \vDash \Psi_{\left(x_{r}, \mathbb{P} z, M_{1} w_{1}, \ldots, M_{s} w_{s}\right)}(\mathbb{P} \delta(y)) \quad$ in $\mathbf{m}$ iff in $\mathbf{m}$ also $x_{r} \vDash \Psi_{\left(x_{r} \diamond z, M_{1} w_{1}, \ldots, M_{s} w_{s}\right)}(\mathbb{P} \delta(y))$.

But this means that at $x_{r}, \diamond \delta(z)=\mathbb{P} \delta(z)$ in $\mathbf{m}$ so $x_{r} \vDash \diamond \delta(z)$ (see Remark 2.15) contrary to our assumption.

Subcase of $\mathbb{P} \quad$ We now examine the case of $\mathbb{P}$. Assume $x_{r} \rho_{1} z$ and assume $x_{r} \vDash \neg \mathbb{P} \delta(z)$ in $\mathbf{m}$. This means that for all $y$ such that $x_{r} \rho_{2} y$ we have $y \vDash \neg \delta(z)$ in $\mathbf{m}$. Hence by the induction hypothesis, $y \vDash \neg \delta(z)$ in $\mathbf{m}_{\left(\pi^{*}, \mathbb{P} y\right)}^{\sharp}$. Hence we have that in $\mathbf{m}_{\pi^{*}}^{\sharp}, x_{r} \vDash \neg \mathbb{P} \delta(z)$. The reason for the last step is that any $y$ such that $x_{r} \rho_{1} y$ is not accessible for $\mathbb{P}$, i.e., such an arc $x_{r} \stackrel{\diamond}{\longrightarrow} y$ is off in $\mathbf{m}^{\sharp}$.

Now assume $x_{r} \rho_{2} z$ in $\mathbf{m}$. Then $x_{r} \vDash \mathbb{P} \delta(z)$ holds in $\mathbf{m}$. Hence $z \vDash \delta(z)$ holds in $\mathbf{m}$. By the induction hypothesis, $z \vDash \delta(z)$ holds in $\mathbf{m}_{\left(\pi^{*}, \mathbb{P} z\right)}^{\sharp}$. If the connection $x_{r} \stackrel{\mathbb{P}}{\longrightarrow} z$ is on in $\mathbf{m}_{\pi^{*}}^{\sharp}$, (i.e., $\mathbf{f}_{\pi^{*}}\left(x_{r} \stackrel{\mathbb{P}}{\longrightarrow} z\right)=1$ ) then we will get that in $\mathbf{m}_{\pi^{*}}^{\sharp}, x_{r} \vDash \mathbb{P} \delta(z)$. 
How can it be the case that $\mathbf{f}_{\pi^{*}}\left(x_{r} \rightarrow z\right)=0$ ? This can happen if along the path $\pi=\left(a, M_{1} x_{1}, \ldots, M_{r} x_{r}\right)$ an odd number of double arrows was triggered towards the $\operatorname{arc} x_{r} \rightarrow z$. To see what this means we go to Case 1 of Definition 2.18. But then let us also consider Remark 2.19 and axiom $\mathbb{R} \mathbb{A}$. We will be in violation of axiom $\mathbb{R} \mathbb{A}$ if an odd number of double arrows hits $x_{r} \rightarrow z$. The axiom says that $x_{r} \rightarrow z$ must be active. This completes the induction case and the Lemma is proved.

We now have almost all that we need for the completeness proof. We basically take axiom $\mathbb{R} \mathbb{A}$ and construct a Henkin type reduced model $\mathbf{m}$, convert it to $\mathbf{m}^{\sharp}$ and use Lemma 2.20.

This we shall do in the next Section 3.

\section{Completeness theorem for KR}

This section provides an axiom system and a completeness theorem for the logic $\mathbf{K R}$ as defined semantically in Definition 2.1. Our strategy is as follows:

Given a formula $A$, we want to construct a model for it. $A$ has a finite number of atoms and no more than $n$ nested modalities, and so we expect to construct a finite model of level $n$ with an assignment to the atoms of $A$. We need to find the right axioms which will enable us to carry out the construction of the correct model.

We begin by syntactically defining all finite models. This is done in Definition 3.1. We also define syntactically a natural system of path nominals for each model and using it we can talk syntactically about paths $\pi$ and their associated paths $\pi^{*}$.

Armed with this syntactical capability, we can write Axiom 3 of Definition 3.4. The formulation of this axiom was discussed at length on page 35 in part 3 of Remark 2.19.

We mention that this axiomatisation is brute force (see discussion below in Remark 3.7), we cannot get around it without possibly adding more connectives.

The reader should note that there is a difference of culture here between the pure mathematical logician and the applied logician. The pure logician would tend to axiomatise with minimum number of connectives and minimum number of variables used. The Polish school of logic, for example, is well known for its minimal axiomatisations of classical logic and its variants, an ongoing activity which was slowed down only by the Second World War.

The applied logician would be more inclined to add connectives and constants as long as they have a natural meaning in the application area. We shall address this possibility in the concluding section.

\subsection{Methodological presentation of the axioms for $\mathbf{K R}$}

Definition 3.1 (Syntactical models) Let $\mathbb{Q}=\left\{q_{1}, \ldots, q_{k}\right\}$ be a set of atoms and let $S$ be an infinite set of names of possible worlds. We shall define inductively the family of all $n$-level syntactical models together with their possible world realisation and a path nominal function $\delta$ for each such model.

1. Any conjunctive normal form of the form

$$
\mu_{0}=\varepsilon=\bigwedge_{q \in \mathbb{Q}} \pm q
$$


is a syntactical level 0 model. Fore each such $\varepsilon$ let $\mathbf{n}_{\varepsilon}=\left(\left\{t_{\varepsilon}, h\right)\right.$ be a corresponding level 0 model, with $t_{\varepsilon} \in S$ and $h\left(t_{\varepsilon}, q\right)=1$ iff $\varepsilon \vdash q$. Assume $\varepsilon \neq \varepsilon^{\prime} \Rightarrow t_{\varepsilon} \neq t_{\varepsilon^{\prime}}$. Let $\delta\left(t_{\varepsilon}\right)=\varepsilon$.

2. The syntactical models of level 1 have the form

$$
\mu=\mu_{1}=\varepsilon_{1} \wedge \bigwedge_{\varepsilon \in I} \diamond \varepsilon \wedge \bigwedge_{\eta \in J} \mathbb{P} \eta \wedge \bigwedge_{\varepsilon \notin I} \neg \vee \varepsilon \wedge \bigwedge_{\eta \notin J} \neg \mathbb{P} \eta
$$

where $\varepsilon_{1}$ is a level 0 syntactical model and $\varepsilon, \eta$ range over level 0 syntactical models and $J, I$ are the sets of level 0 syntactical models.

The corresponding level 1 reduced model realisation of $\mu_{1}$ is defined as follows

$$
\mathbf{n}_{\mu}=\left(S_{\mu}, \rho_{1}, \rho_{2}, a_{\mu}, h\right)
$$

where

$$
S_{\mu}=\left\{a_{\mu}\right\} \cup\left\{t_{\varepsilon} \mid \varepsilon \in I\right\} \cup\left\{s_{\varepsilon} \mid \varepsilon \in J\right\} .
$$

We assume $a_{\mu}, t_{\varepsilon}, s_{\varepsilon}$ are all different elements of $S$.

We let $a_{\mu} \rho_{1} t_{\varepsilon}, \varepsilon \in I$ and $a_{\mu} \rho_{2} s_{\varepsilon}, \varepsilon \in J$.

Let

$$
\begin{aligned}
& h(a, q)=1 \text { iff } \varepsilon_{1} \vDash q \\
& h\left(t_{\varepsilon}, q\right)=1 \text { iff } \varepsilon \vDash q \\
& h\left(s_{\varepsilon}, q\right)=1 \text { iff } \varepsilon \vDash q
\end{aligned}
$$

Let

$$
\begin{aligned}
& \delta\left(t_{\varepsilon}\right)=\varepsilon \\
& \delta\left(s_{\varepsilon}\right)=\varepsilon \\
& \delta\left(a_{\mu}\right)=\mu
\end{aligned}
$$

3. Assume the syntactical models are defined for any level $\leq m$. Also assume that for any such syntactical model $\mu$ a realisation model $\mathbf{n}_{\mu}=\left(S_{\mu}, \rho_{1}, \rho_{2}, a_{\mu}, h\right)$ is also defined with

$$
\mu_{1} \neq \mu_{2} \Rightarrow S_{\mu_{1}} \cap S_{\mu_{2}}=\oslash
$$

Let $I, J$ be any sets of syntactical models of level $\leq m$. Assume at least one model in $I \cup J$ is of level $m$ and not of any level $<m$.

Define the level $m+1$ model $\mu$ as follows

$$
\mu=\varepsilon_{\mu} \wedge \bigwedge_{\alpha \in I} \nabla \alpha \wedge \bigwedge_{\beta \in J} \mathbb{P}_{\beta} \wedge \bigwedge_{\alpha \notin I} \neg \nabla \alpha \wedge \bigwedge_{\beta \notin J} \neg \mathbb{P}_{\beta} .
$$

Define the level $m+1$ realisation model for $\mu$ as follows:

$$
\mathbf{n}_{\mu}=\left(S_{\mu}, \rho_{1}, \rho_{2}, a_{\mu}, h\right) .
$$

For each $\alpha \in I, \beta \in J$ let $\mathbf{n}_{\alpha}=\left(S_{\alpha}^{i}, \rho_{1}^{\alpha}, \rho_{2}^{\alpha}, a_{\alpha}, h_{\alpha}\right)$ and $\mathbf{n}_{\beta}=\left(S_{\beta}^{J}, \rho_{1}^{\beta}, \rho_{2}^{\beta}, a_{\beta}, h_{\beta}\right)$ be their respective realisation models. Assume all $S_{\alpha}^{I}, S_{\beta}^{J}$ are pairwise disjoint. Also assume $\delta_{\alpha}, \delta_{\beta}$ are given on $\mathbf{n}_{\alpha}, \mathbf{n}_{\beta}$. Let $S_{\mu}=\left\{a_{\mu}\right\} \cup \bigcup_{\alpha \in I} S_{\alpha}^{I} \cup \bigcup_{\beta \in J} S_{\beta}^{J}$ where $a_{\mu}$ is a new label from $S$. 
Define

$$
\begin{aligned}
& a_{\mu} \rho_{1} a_{\alpha}, \alpha \in I \\
& a_{\mu} \rho_{2} a_{\beta}, \beta \in J
\end{aligned}
$$

$\rho_{i}, i=1,2$ is the same as $\rho_{i}^{\alpha}$ on $S_{\alpha}^{I}$, for $\alpha \in I$ and $\rho_{i}$ be the same as $\rho_{\beta}^{J}$ for $\beta \in J$. Let $h\left(a_{\mu}, q\right)=1$ iff $\varepsilon_{\mu} \vDash q$ and let $h$ be $h_{\alpha}$ on $S_{\alpha}^{I}$ and $h_{\beta}$ on $S_{\beta}^{J}$.

Let $\delta_{\mu}$ be $\delta_{\alpha}$ on $S_{\alpha}^{I}, \delta_{\beta}$ on $S_{\beta}^{J}$ and let $\delta\left(a_{\mu}\right)=\mu$.

Lemma 3.2 Let $\mu$ be a syntactical model and let $\mathbf{n}_{\mu}$ be its realisation as defined in Definition 3.1. Let $\delta_{\mu}$ be its path nominal system as defined in Definition 3.1.

Then $\delta_{\mu}$ is indeed a path nominal function according to Definition 2.4 and Lemma 2.14 holds for $\delta_{\mu}$.

Proof Simple, by induction.

Lemma 3.3 Let $\mathbf{m}=\left(S, \rho_{1}, \rho_{2}, a, h\right)$ be a reduced model of level $n$ for $\mathbb{Q}$. Let $\mu$ be a syntactical model for $\mathbb{Q}$. Assume that $a \vDash \mu$. Let $\mathbf{n}_{\mu}$ be the realisation model for $\mu$. Then $\mathbf{m}$ is isomorphic with $\mathbf{n}_{\mu}$.

Proof By induction on the level of $\mathbf{m}$.

Definition 3.4 (Axiom system for KR) Consider the following axioms for KR.

1. Modal $K$ axioms and rules for $\mathbb{P}$ and $\diamond$.

2. $\mathbb{P} A \rightarrow \diamond A$ for $A$ not containing $\mathbb{P}$.

3. For each syntactical model $\mu$, let $\mathbb{R} \mathbb{A}(\mu)$ be the axiom as defined on page 35 in Part 3 of Remark 2.19. Then our axiom is $\mu \rightarrow \mathbb{R} \mathbb{A}(\mu)$.

Remark 3.5 Note that by axiom (2) $\mathbb{P} q \rightarrow \diamond q$ is a theorem for atomic $q$. Thus $\mu=$ $q \wedge \mathbb{P} q \wedge \neg \vee q$ is an inconsistent level 1 syntactical model for $\{q\}$. This does not affect Axiom 3 because $\mu$ is in the antecedent!

Remark 3.6 Let us comment on Axiom 2 of our logic:

1. If $A$ is a wff without $\oslash$, then $\mathbb{P} A \rightarrow \diamond A$. This is not true for arbitrary $A$. Consider Fig. 27.

In this figure, $s \rightarrow r$ is off, so $\diamond \mathbb{P} q$ is false at $t$, however, $\mathbb{P P} q$ is the true at $t$, because the double arrow $(t \rightarrow s) \rightarrow(s \rightarrow r)$ is triggered by $\mathbb{P}$ and so it activates the connection $s \rightarrow r$ and hence $s \vDash \mathbb{P} q$.

Remark 3.7 The perceptive reader will have noticed that our axiomatisation of $\mathbf{K R}$ is a 'brute-force' axiom system. Our 'axioms' simply describe syntactically the correct semantics. Perhaps the reader would have expected a nicer axiom system. This is not possible. We shall offer an explanation by giving an example.

Consider ordinary modal logic $\mathbf{K}$ with a single modality $\diamond$. Its models have the form $(S, R, a), a \in S, R \subseteq S \times S$ and $R$ is not necessarily reflexive. The truth condition for $\diamond$ is (1).

1. $t \vDash \diamond A$ iff for some $s, t R s$ and $s \vDash A$. 


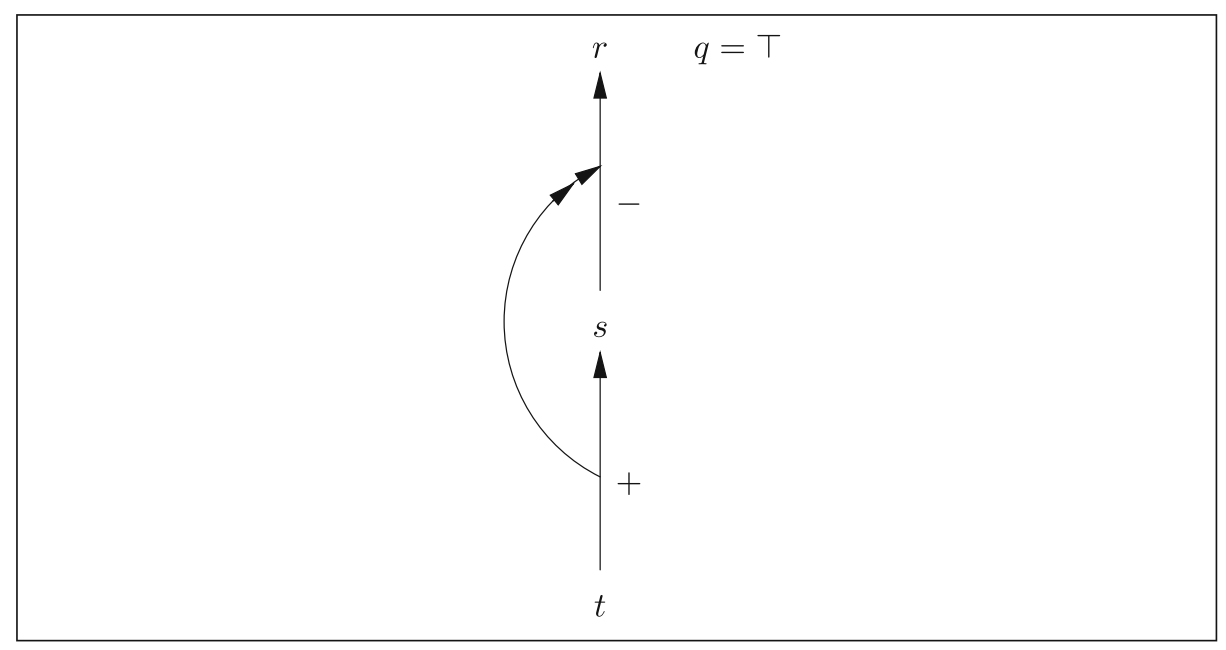

Fig. 27 Illustrating Remark 3.6

Let us now change the semantics a bit. Consider models of the form $(S, E, R, a)$, with $E \subseteq S$. We change the truth condition to be

2.

$$
t \vDash \diamond A \text { iff }\left\{\begin{array}{l}
t \notin E \text { and for some } s, t R s \text { and } s \vDash A \\
\text { and } \\
t \in E \text { and } t \vDash A \text { or for some } s, t R s \text { and } s \vDash A
\end{array}\right.
$$

Thus the set $E$ tells us that we want reflexivity.

Now define a family of finite models $(S, E, R, a, h)$ as follows.

Take any finite model $\mathbf{m}=(S, R, a, h)$. Code this model by a Turing machine. Apply an effective procedure to extract a set $E_{\mathbf{m}} \subseteq S$, and define the new model $\mathbf{n}=\left(S, E_{\mathbf{m}}, R, a, h\right)$.

Let the logic $\mathbf{K}_{R}$ be defined by the set of all such model $\mathbf{n}$.

Now I ask the reader how can you axiomatise this logic without syntatically describing the models $\mathbf{n}$ ?

If the definition of $\mathbf{n}$ is simple we can probably do it nicely but otherwise bruteforce is needed.

For example, we can let

$$
t \in E \text { iff } \exists x(x R t \wedge \neg x R x) .
$$

Then maybe we can axiomatise this logic nicely.

In the case of KR we allow double arrows to go anywhere and we can switch connections on and off. This is too free to control nicely, so brute-force is called for. Our difficulty is compounded by the fact that we do not have any modality or other special connective controlling the double arrows, so our only means to describe their behaviour is through the coding of the changes they cause in the model, hence the need for a brute force axiomatisation.

Here are some simple conditions on reactivity which can make for nicer logics. 


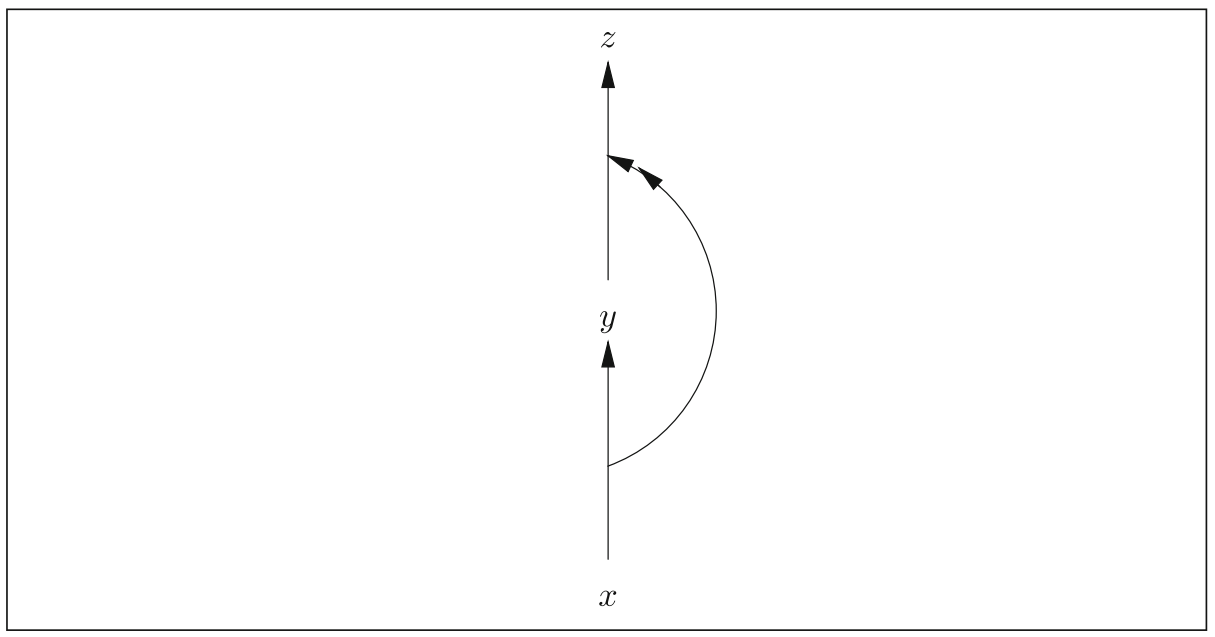

Fig. 28 Next step reactivity

Next step reactivity If $(x \rightarrow y) \rightarrow(w \rightarrow z) \in R_{2}$ then $y=w$. I.e. we are allowed double arrows only in the form of Fig. 28

Forward look reactivity The target of the next arrow must be above the target of a previous arrow. Namely if

$$
(x \rightarrow y) \rightarrow(u \rightarrow v) \in R_{2}
$$

and

$$
\left(x^{\prime} \rightarrow y\right) \rightarrow\left(u^{\prime} \rightarrow v^{\prime}\right) \in R_{2}
$$

and for some $m \geq 1$

$$
x \rho^{m} x^{\prime}
$$

Then for some $n$

$$
u \rho^{n} u^{\prime}
$$

In words: a later arrow must 'hit' a later target.

Figure 29 illustrates the idea.

\subsection{The canonical model}

We now construct a canonical finite reduced model for two modalities $\mathbb{P}$ and $\diamond$ and then covert it to a KR model. We shall construct a finite model for any consistent formula $A$. This will also give us finite model property and decidability.

Our starting point is to construct a very specific bi-modal finite model with two accessibility relations, one for $\square$ and the other for $\oslash$. Additional axioms will allow us to convert this special model into a reactive one.

We need some definitions.

1. A wff $A$ is said to be of level $n$ if $n$ is the maximal number of nested $\{\mathbb{P}, \diamond\}$ in $A$. 


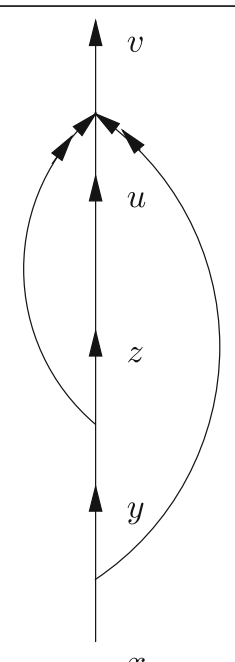

$x$

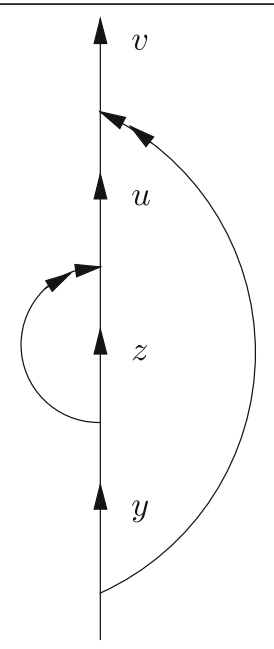

$x$

Fig. 29 A later arrow 'hit' a later target

2. Let $\mathbb{Q}=\left\{q_{1}, \ldots, q_{k}\right\}$ be a set of atoms, then let $\mathbb{Q}^{n}$ be the set of all wffs based on $\mathbb{Q}$ of level $\leq n$.

Let $A$ be a consistent wff. We want to construct a finite model for $A$. Let $q_{1}, \ldots, q_{k}$ be all atomic sentences appearing in $A$ and let $n$ be the maximal number of nested modal operators $\{\mathbb{P}, \diamond\}$ in $A$.

Step 1 We first extend $A$ to a theory $\Theta$ containing $A$ satisfying the following conditions

1. For any wff $B$ built up from $\left\{q_{1}, \ldots, q_{k}\right\}$ and containing no more than $n$ nested modalities we have $B \in \Theta$ or $\neg B \in \Theta$

2. $\Theta$ is $\mathbf{K R}$ consistent. We shall construct a finite model for $\Theta$.

Step $2 a$ Let $\mathbb{P} D \in \Theta$.

We say $E_{D}$ of level $\leq n-1$ is maximal for $\mathbb{P} D \in \Theta$, iff $\mathbb{P}\left(D \wedge E_{D}\right) \in \Theta$ and whenever $\mathbb{P} x \in \Theta$ and $X$ is of level $\leq n-1$ and $\vdash D \wedge E_{D}$. We also have $D \wedge E_{D} \vdash X$.

We now show that such an $E_{D}$ exists.

Lemma 3.8 Let $\mathbb{P} Y \in \Theta$ and let $X$ be any wff of level $\leq n-2$. Then either $\mathbf{P}(Y \wedge$ $X) \in \Theta$ or $\mathbb{P}(Y \wedge \neg X) \in \Theta$.

Proof If neither is in $\Theta$ then $\neg \mathbb{P}(Y \wedge X) \wedge \neg \mathbb{P}(Y \wedge \neg X)$ is in $\Theta$. Therefore

$$
\oslash(Y \rightarrow \neg X) \wedge \oslash(Y \rightarrow X) \in \Theta
$$

and hence $\oslash \neg Y \in \Theta$ contradicting the consistency of $\Theta$. 
From the lemma it follows that we can add inductively $\pm X_{m+1}$ to $\mathbb{P}\left(Y \wedge \pm x_{1} \wedge\right.$ $\left.\ldots \wedge \pm x_{m}\right)$ and hence such a maximal $E_{D}$ exists for any $\mathbb{P} D \in \Theta$.

This is so because assume we added $Y_{i}$ such that $\mathbb{P}\left(Y \wedge \wedge Y_{i}\right) \in \Theta$ and for every $Z$ of level $\leq n-1$, there exists an $i$ such that $Y_{i}=Z$ or $Y_{i}=\neg Z$.

Now assume $\mathbb{P} U \in \Theta$ and $U \vdash Y \wedge \wedge Y_{i}$.

Since $u$ is of level $\leq n-1$ then for some $Y_{i}$, either $Y_{i}=U$ or $Y_{i}=\neg U$. If $Y_{i}=U$ then $Y \wedge \wedge_{i} Y_{i} \vdash U$ as required.

If $Y_{i}=\neg U$ then we get that $U \vdash \neg U$, i.e., $U \vdash \perp$ and so $\mathbb{P} \perp \in \Theta$. This is not possible.

Let $\mathbb{P}\left(D^{i} \wedge E_{D}^{i}\right) \in \Theta$ be the entire list of all such maximal wffs, $i=1, \ldots, r_{n}$. Note that the index ' $n$ ' in ' $r_{n}$ ' is the level $n$ of the theory $\Theta$. In the sequel we shall use theories of level $n-m, m=1,2, \ldots$ and we shall respectively use $r_{n-m}, m=1,2 \ldots$ Similarly we shall introduce below $s_{n}, s_{n-1} \ldots$

Step $2 b$ Similarly suppose we have that $\diamond B \in \Theta$, then there exists a maximal $E_{B}$ such that $\diamond\left(B \wedge E_{B}\right) \in \Theta$.

We need another lemma.

Lemma 3.9 Let $\diamond B \in \Theta$ and let $x$ be any wff of level $\leq n-1$. Then either $\nabla(B \wedge X) \in$ $\Theta$ or $\diamond(B \wedge \neg X) \in \Theta$. Otherwise

$$
\neg \diamond(B \wedge X) \wedge \neg \diamond(B \wedge \neg X \in \Theta
$$

hence $\square(B \rightarrow X) \wedge \square(B \rightarrow \neg X) \in \Theta$ and hence $\square \neg B \in \Theta$ contradicting the consistency of $\Theta$.

We can thus let $\diamond\left(B_{j} \wedge E_{B_{j}}\right) \in \Theta$ enumerate all the wffs of this form which are maximal and are in $\Theta$, for $j=r_{n}+1, \ldots, r_{n}+s_{n}$.

Lemma 3.10 If $\diamond X \in \Theta$ and $\diamond Y \in \Theta$ and $X$ and $Y$ are maximal then either $X \equiv Y$ or $(X \forall Y$ and $Y \nvdash X)$.

Proof If $Y \vdash X$ then $X$ and $Y$ are consistent. We get by maximality of $X$ that $X \vdash Y$, hence $X \equiv Y$. Similarly for $\mathbb{P} X, \mathbb{P} Y$.

The above two step considerations proved the following.

Lemma 3.11 Let $\mathbb{Q}$ be finite and let $\Theta$ be any complete theory of the language $\mathbb{Q}^{n}$.

Then there exists $D_{1}^{n-1}, \ldots, D_{r_{n}}^{n-1}, B_{r_{n}+1}^{n-1}, \ldots, B_{r_{n}+s_{n}}^{n-1}$ of level $\leq n-1$ such that the following holds

1. $\mathbb{P} D_{i}^{n-1} \in \Theta, i=1 \ldots r_{n}$

2. $\diamond B_{j}^{n-1} \in \Theta, j=r+n+1, \ldots, r_{n}+s_{n}$

(a) $\mathbb{P} C_{k}^{a-1} \wedge \diamond C_{k}^{n-1} \in \Theta, s_{n}+1 \leq k \leq w_{n}$

3. For any $D$ such that $D \in \Theta$, there exists an $i$ such that $D_{i}^{n-1} \vdash D$.

4. For any $B$ such that $\diamond B \in \Theta$, there exists a $j$ such that $B_{j}^{n-1} \vdash B$.

5. For any two non equivalent $Y, X \in\left\{D_{i}^{n-1}, B_{j}^{n-1}\right\}$ we have $Y \nvdash X$ and $X \nvdash Y$. 
Step 3 Consider the situation of Lemma 3.11.

1. For each $\mathbb{P} D_{i}^{n} \in \Theta$ let $\Delta_{i}^{n-1}=\left\{D_{i}^{n-1}\right\} \cup\{\mid U$ of level $\leq n-1$ and $\varnothing U \in \Theta\}$. This set is consistent and can be extended to a complete theory $\Theta_{i}^{n-1}$ in the language $\mathbb{Q}^{n-1}$.

2. Similarly for any $\diamond B_{j}^{n-1} \in \Theta$ we let $\Delta_{j}^{n-1}=\left\{B_{j}^{n-1}\right\} \cup\{U \mid U$ of level $\leq n-1$ and $\square U \in \Theta\}$.

This set is consistent and can be extended to a complete and consistent theory $\Theta_{j}^{n-1}$ of $\mathbb{Q}^{n-1}$.

Step 4 We start with a complete and consistent theory $\Theta$ of $\mathbb{Q}^{n}$ and construct a finite tree model for it as follows. $\left(S, R, \rho_{\diamond}, \rho_{\mathbb{P}}\right)$ is the tree. We first define $S$ and $R$ in steps.

Construction step 4.0 Let $0 \in S$ and let $\Delta_{0}=\Theta$.

Construction step 4.1 Consider the situation described in Lemma 3.11 for $\Theta$. Let $(0, i), i=1, \ldots, r_{n}+1$ be new elements which we put into $S$ and let

$$
\begin{aligned}
& \Delta_{(0, i)}=\Theta_{i}^{n-1}, i=1, \ldots, r_{n} \\
& \Delta_{(0, j)}=\Theta_{j}^{n-1}, j=r_{n}+1, \ldots, r_{n}+s_{n}
\end{aligned}
$$

Construction step $4 . m$ for $1<m \leq n$ Assume $S$ contains a sequence $\mathbf{t}=$ $\left(0, x_{1}, \ldots, x_{m}\right)$ and that a theory $\Theta^{n-m}=\Delta_{\left(0, x_{1}, \ldots, x_{m}\right)}^{n-m}$ has been defined.

We repeat the construction of step 1 for the theory $\Theta^{n-m}$ using the indices $r_{n-m}$ and $s_{n-m}$ and define respective sequences

$$
\mathbf{t} *(i), i=1, \ldots, r_{n-m}
$$

and

$$
\mathbf{t} *(j),=r_{n-m}+1, \ldots, r_{n-m}+s_{n-m}
$$

and put them in $S$. We also define theories $\Delta_{\mathbf{t} *(i)}^{n-m-1}$ and $\Delta_{\mathbf{t} *(j)}^{n-m-1}$ using $\Theta_{i}^{n-m}$ and $\Theta_{j}^{n-m}$ respectively as done in Step 4.1.

The construction stops at $m=n-1$. We get a set $S$ of sequences $\mathbf{t} \in S$. The set forms a tree under the relation $\mathbf{t} R \mathbf{s}$ iff $\exists x(\mathbf{s}=t *(x))$. With each $\mathbf{t}$ of length $m$ we have a theory $\Delta_{\mathbf{t}}^{n-m}$.

For $\mathbf{t}=\left(0, x_{1}, \ldots, x_{m}\right)$ let $\mathbf{t} \rho_{\mathbb{P}} \mathbf{s}$ hold when $\mathbf{s}=\mathbf{t} *(i)$ for $1 \leq i \leq r_{n-m}$ (i.e. $\mathbf{s}$ was created because of $\mathbb{P} * D_{i}^{n-m}$ in $\left.\Delta_{\mathbf{t}}^{n-m}\right)$. $\mathbf{t} \rho \diamond \mathbf{s}$ iff $\mathbf{s}=\mathbf{t} *(j)$ for $r_{n-m}+1 \leq j \leq s_{n-m}$ (i.e., $s$ was created because of $\diamond B_{j}^{n-m} \in \Theta$ ).

Define an assignment $h$ as follows: $\mathbf{t} \vDash q$ iff $q \in \Delta_{\mathbf{t}}$.

Lemma 3.12 In the model $\left(S, R, \rho_{\diamond}, \rho_{\mathbb{P}}, a, h\right)$ we have for any wff of level $\leq n-m$ and any $\mathbf{t}$ of length $m$

$$
\mathbf{t} \vDash A \text { iff } A \in \Delta_{\mathbf{t}} .
$$

Proof By induction on $A$

For atoms this holds. 
Consider the case of $\mathbb{P} A$.

1. Assume $\mathbb{P} A \in \Delta_{\mathbf{t}}^{n-m}$. Then for some $D_{i}^{n-m-1}$ we have $D_{i}^{n-m-1} \vdash A$ and $\mathbb{P} D_{i}^{n-m-1} \in \Delta_{\mathbf{t}}^{n-m}$. Hence $D_{i}^{n-m-1} \in \Delta_{\mathbf{t} *(i)}^{n-m-1}$. By the induction hypothesis $\mathbf{t} *(i) \vDash$ $D_{i}^{n-m-1}$ and therefore $\mathbf{t} *(i) \vDash A$.

2. Assume $\oslash A \in \Delta_{\mathbf{t}}^{n-m}$. Then by construction for any $i, 1 \leq i \leq r_{n-m}$ we have $A \in$ $\Delta_{\mathbf{t} *(i)}^{n-m-1}$ and so $\mathbf{t} *(i) \vDash A$, and hence $\mathbf{t} \vDash \oslash A$.

The proof for $\diamond$ is similar.

We have proved weak completeness for the logic with $\diamond$ and $\mathbb{P}$ using the axioms of $\mathbf{K}$ for each.

We got $R=\rho_{\diamond} \cup \rho_{\mathbb{P}}$ and we need to covert this model to a model of KR with $R_{1}$ and $R_{2}$.

We now need to use our additional axioms $\mathbb{R} \mathbb{A}$ to determine how $\rho_{\mathbb{P}}$ is correctly derived from a set of double arrows $R_{2}$.

Remark 3.13 We make some observations about the model $\left(S, R, \rho_{\diamond}, \rho_{\mathbb{P}}, 0, h\right)$ which we constructed.

Let $\mathbf{t}_{m}=\left(0, x_{1}, \ldots, x_{m}\right)$ be a point in the tree. Let $t_{i}=\left(0, x_{1}, \ldots, x_{i}\right), i \leq m$. We know $\mathbf{t}_{i+1}=\mathbf{t}_{i} *\left(x_{i+1}\right)$.

1. If $x_{i+1} \leq r_{i}$ then $\mathbb{P} D_{x_{i}}^{n-i-1} \in \Delta_{\mathbf{t}_{i}}^{n-1}$ and $D_{x_{i}}^{n-i-1} \in \Delta_{\mathbf{t}_{i+1}}^{n-i-1}$. We write $x_{i} \stackrel{\mathbb{P}}{\longrightarrow} x_{i+1}$.

2. If $r_{i}<x_{i+1} \leq s_{i}$ then $\diamond B_{x_{i}}^{n-i-1} \in \Delta_{\mathbf{t}_{i}}^{n-i}$ and $B_{x_{i}}^{-i-1} \in \Delta_{t_{i+1}}^{n-i-1}$. We write $x_{i} \stackrel{\diamond}{\longrightarrow} x_{i+1}$.

Let $E_{x_{i}}$ be the key formula $D_{x_{i}}^{n-i-1}$ or $B_{x_{i}}^{n-i-1}$ as the case may be. Then clearly at $\Delta_{0}^{n}$ the following holds

$$
\beta_{\mathbf{t}_{m}}=M_{1}\left(E_{x_{1}} \wedge M_{w}\left(E_{x_{2}} \wedge \ldots M_{m-1}\left(E_{x_{m-1}} \wedge M_{m} E_{x_{m}}\right) \ldots\right)\right.
$$

where $M_{i} \in\{\mathbb{P}, \diamond)$ and $M_{i}=\mathbb{P}$ if $\leq x_{i} \leq r_{i}$ and $M_{i}=\diamond$ if $r_{i}+1 \leq x_{i} \leq s_{i}$.

We write $x_{i} \stackrel{M_{i}}{\longrightarrow} x_{i+1}$.

Clearly by the completeness theorem Lemma 3.12, we also have $0 \vDash \beta_{\mathbf{t}_{m}}$.

Following Lemma $3.10 \beta_{\mathbf{t}_{m}}$ uniquely characterises the path leading to $\mathbf{t}_{m}$ in the model. Therefore $\delta_{x}^{*}=E_{x}$, as defined in Lemma 3.11, can act as path nominals for the points $\mathbf{t}$ via their validity at 0 .

Theorem 3.14 (Completeness theorem for KR) KR of Definition 3.4 is complete for the proposed semantics of Definition 2.1.

Proof Let $A$ be a consistent formula. We constructed reduced finite bi-modal model m for $A$ as in Section 3.2. By Lemma 3.12, $A$ holds in this model. By Remark 2.19 and Lemma 2.20, $\mathbf{m}$ can be converted to a KR model in which $A$ holds.

Theorem 3.15 (Decidability ad finite model property) KR has the finite model property and is decidable.

Proof From Theorem 3.14. 


\section{Concluding remarks}

We saw that reactive models are very useful and the idea of reactivity is applicable in many areas. The basic system KR of modal reactivity turned out to be not so easily axiomatisable and this calls for possibly some simplifying assumptions on the reactive double arrows, possibly emerging from applications.

So this is one direction of future research.

Another direction is to look at some of the new additional modal connectives and use them to introduce reactivity. We chose $\diamond$ and $\mathbb{P}$, where $\diamond$ ignores reactivity and $\mathbb{P}$ uses it. This is the most direct and natural connective. However, we can approach reactivity 'sideways' as can be seen from Fig. 30.

Since our models are trees, the node $x_{i+1}$ completely identifies the arc $x_{i} \rightarrow x_{i+1}$. Similarly, the node $v$ completely identifies $u \rightarrow v$. Thus if we add a new binary relation $R_{3}$ on $S$ with $v R_{3} x$ reading

- There is a double arrow of the form ((predecessor of $x) \rightarrow x) \rightarrow(($ predecessor of $v) \rightarrow v$ )

We can map all double arrows this way.

So in a reactive model, $R_{2}$ can be represented by $R_{3}$. So we can have a new modality say $\mho^{\prime}$ for double arrows. We can add a special proposition on and have

$$
v \vDash \diamond^{\prime} \text { on iff } \exists x\left(v R_{3} x \wedge x \vDash \text { on }\right)
$$

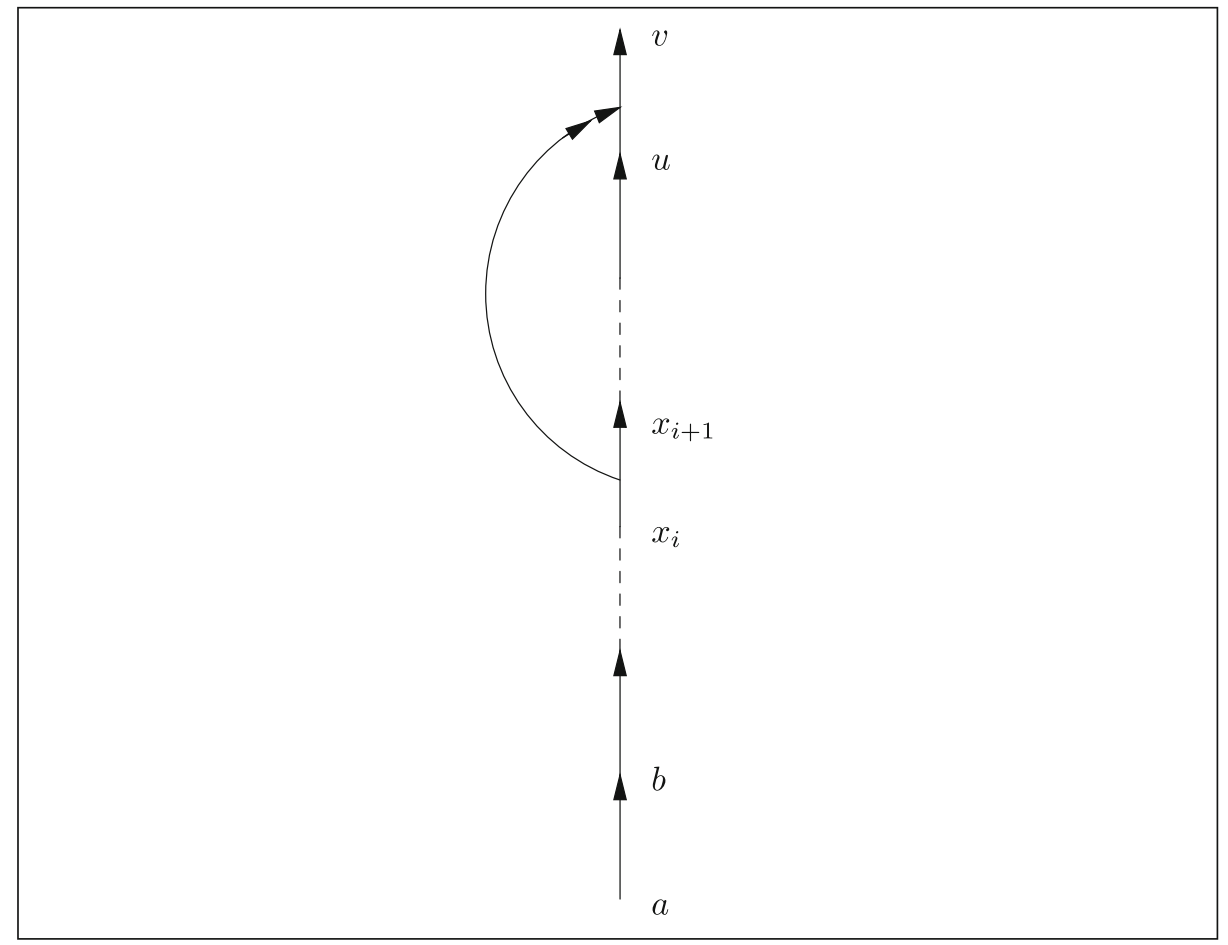

Fig. 30 Reducing reactivity to modality in case of trees 
This device may give us some scope for simplifying the axiomatisation but we still need to represent the switch nature of the double arrows. We still have to count how many 'hits' $v$ receives, and it is this part which makes the axiomatisation complicated.

We invite the reader to use the classical translation of modal logic into classical logic. In classical logic we have the full power of the classical quantifiers and still it is not simple to express that $\left\{y \mid x R_{3} y\right\}$ is odd.

Furthermore, $\diamond$ can ignore the double arrows and so we need to count the 'hits' from arcs not ignored by $\diamond$. 'Ignored by $\diamond$ ' implies 'motion' along the arcs and this is foreign to traditional modal logic or to classical logic.

My guess is that axiomatising with the aid of $\diamond^{\prime}$ or any additional tricks would still be very complicated.

Of course good restrictions on the reactivity can simplify the system considerably, and with good luck the simplified system may still be interesting and applicable. One such restriction is the next step reactivity, mentioned in Remark 3.7.

Proposition 4.1 Any next step reactivity logic system is equivalent to a bi-modal system with one modality implying the other!

Proof Figure 31 explains the idea of the proof.

So that is simple enough! It is also still more powerful. Theorem 1.2 still holds.

So we need to find good conditions on $R_{1}$ and $R_{2}$ of the semantics! Indeed, there is still lots to be done.

Remark 4.2 The idea of modality $\nabla^{\prime}$ and its corresponding relation $R_{3}$ has other unexpected consequences. Consider the situation in Fig. 32. This is an interesting situation where we have a higher level double arrow of the form $(b \rightarrow c) \rightarrow$ $((a \rightarrow b) \rightarrow(c \rightarrow d)$.

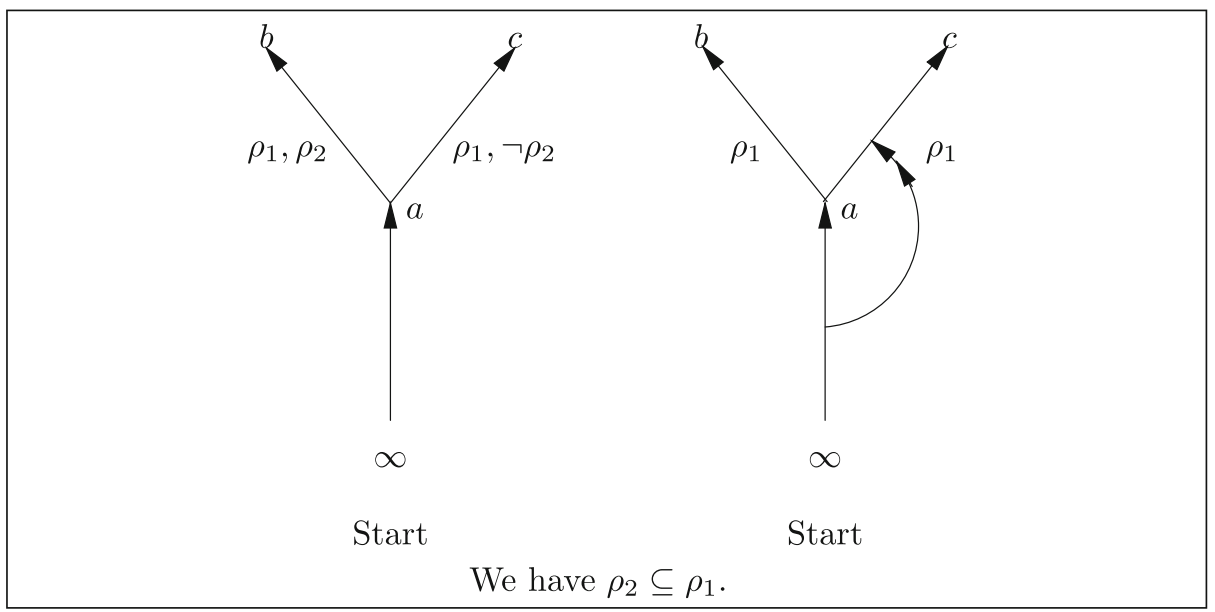

Fig. 31 Illustrating the idea of Proposition 4.1 


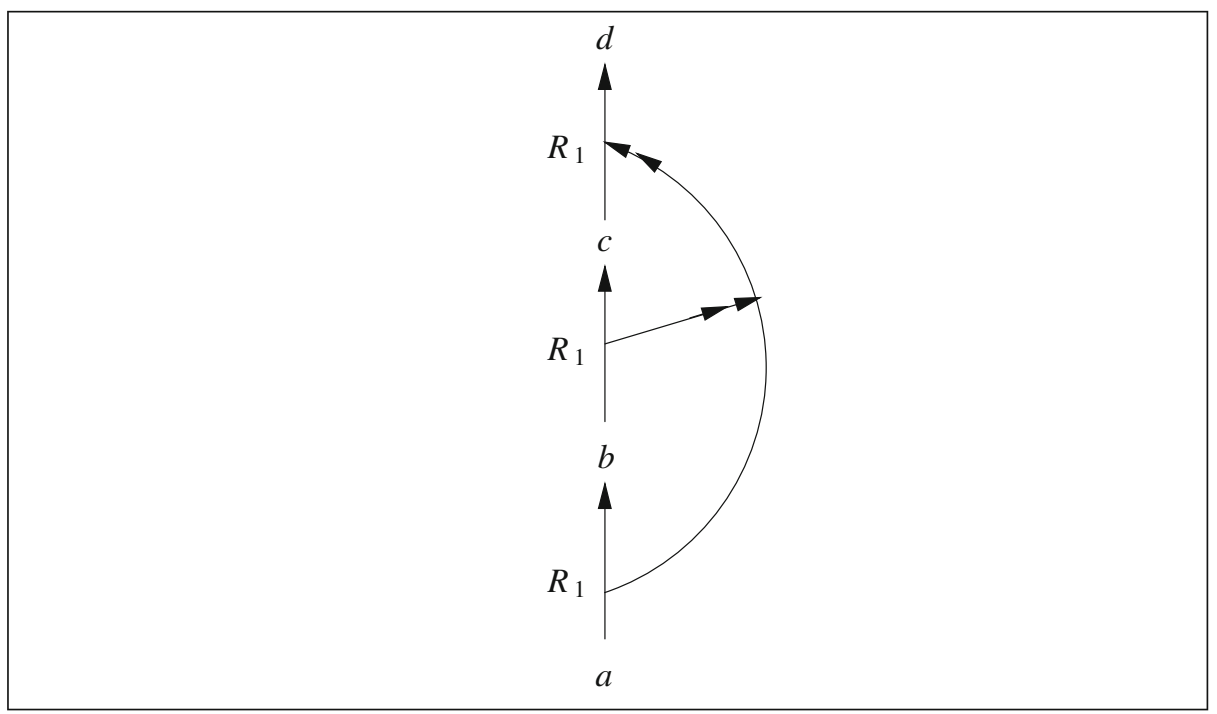

Fig. 32 Higher level double arrow

If we use the relation $R_{3}$ and the modality $\diamond^{\prime}$, we get the situation in Fig. 33. Now we have a situation with ordinary double arrows, so we can use a new relation $R_{4}$ and a modality $\mho^{\prime \prime}$ to eliminate all double arrows, as in Fig. 34.

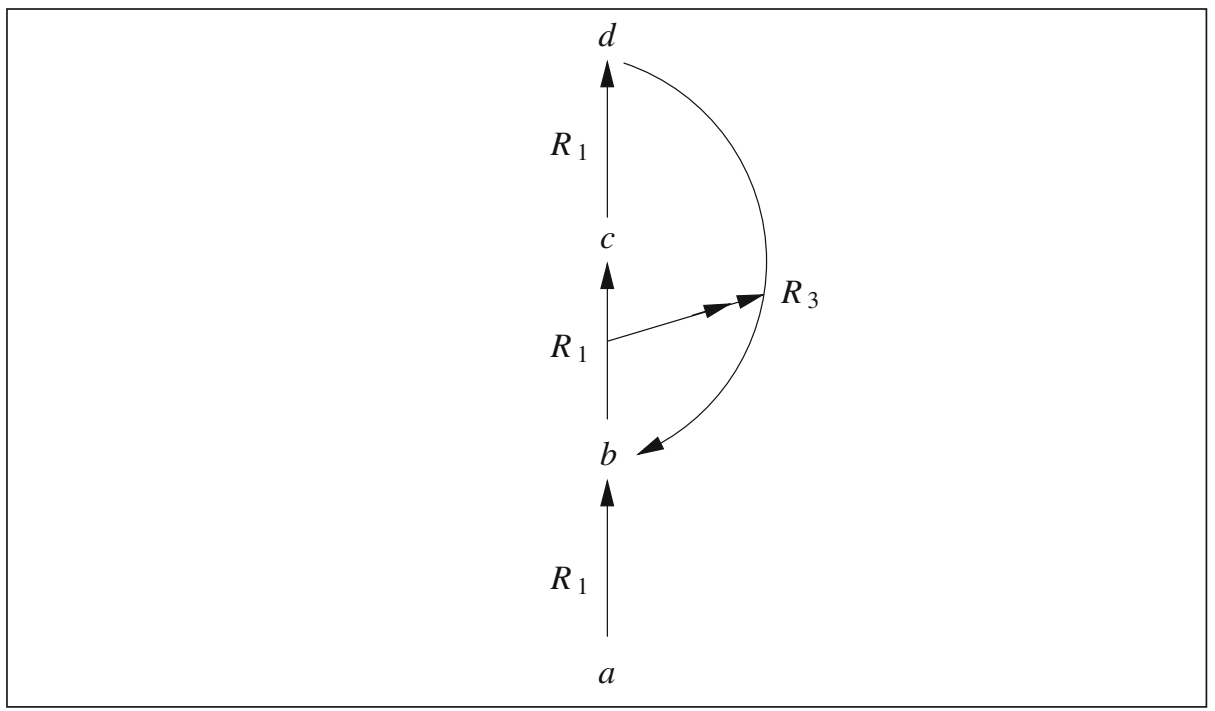

Fig. 33 Reducing Fig. 32 


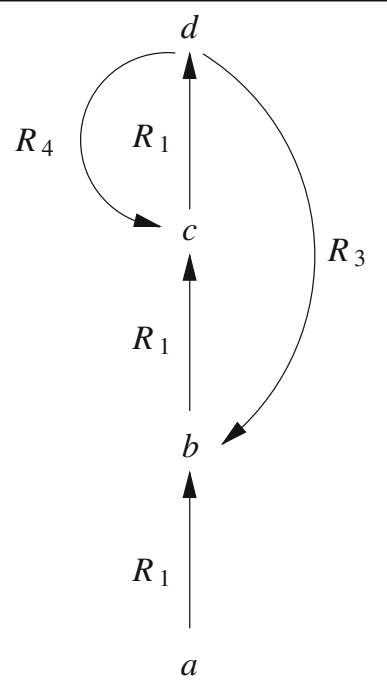

Fig. 34 Reducing Fig. 33

We must remember that $y R_{4} x$ indicates double arrows from the arc ((the $R_{1}$ predecessors of $x) \rightarrow x$ ) to the arc ((the $R_{3}$ predecessor of $\left.y\right) \rightarrow y$ ). So when we have many relations $R$, a better notation would be as follows:

Use $R_{R_{1}, R_{1}}$ instead of $R_{3}$.

Use $R_{\left(R_{1}\left(R_{1}, R_{1}\right)\right)}$ instead of $R_{4}$.

The conclusion from this example is that higher level double arrows can be reduced to multiple modalities. Looking at it the other way round, (in the spirit of Proposition 4.1) multimodal logic can be reduced (given the right conditions) to a higher level reactive modal logic with one modality.

These investigations we postpone for later.

\section{References}

1. Abraham, M., Gabbay, D., Belfer, I., Schild, U.: Future determination of entities in Talmudic logic. Journal of Applied logic (2012, to appear). doi:10.1016/j.jal.2012.06.001G

2. Crochemore, M., Gabbay, D.M.: Reactive automata. Inf. Comput. 209(4), 692-704. doi: 10.1016/j.ic.2011.01.002

3. Gabbay, D.: Reactive Kripke semantics. In: Proceedings of CompLog 2004, pp. 7-20. Centre of Logic and Computation, University of Lisbon (2004)

4. Gabbay, D.: Introducing reactive modal tableaux. This issue of AMAI (2012)

5. Gabbay, D.M.: Reactive Kripke semantics and arc accessibility. In: Avron, A., Dershowitz, N., Rabinovich, A. (eds.) Pillars of Computer Science*: Essays Dedicated to Boris (Boaz) Trakhtenbrot on the Occasion of His 85th Birthday. Lecture Notes in Computer Science, vol. 4800, pp. 292-341. Springer, Berlin (2008). Revised version in this issue

6. Gabbay, D.M.: Reactive Kripke models and contrary-to-duty obligations. In: van der Meyden, R., van der Torre, L. (eds.) DEON-2008: Deontic Logic in Computer Science. LNAI 5076, pp. 155-173. Springer, Heidelberg (2008) 
7. Gabbay, D.M.: Fibring Logics. OUP (1998)

8. Gabbay, D.M.: Reactive intuitionistic tableaux. In: Beth, E.W., van Benthem, J., Kuipers, T., Visser, H. (eds.) Synthese, Special issue, vol. 179, no. 2, pp. 253-269 (2011) http://www. springerlink.com/openurl.asp?genre=article \&id $=$ doi:10.1007/s11229-010-9781-8

9. Gabbay, D., Barringer, H., Woods, J.: Temporal dynamics of argumentation networks. In: Hutter, D., Stephan, W. (eds.) Volume Dedicated to Joerg Siekmann. Mechanising Mathematical Reasoning, Springer Lecture Notes in Computer Science, vol. 2605, pp. 59-98 (2005)

10. Gabbay, D.M., Barringer, H., Rydeheard, D.: Reactive grammars. In: Dershowitz, N. (ed.) To appear in a LNCS volume in Honour of Yakov Choueka. Springer

11. Gabbay, D.M., Marcelino, S.: Modal logics of reactive frames. Stud. Log. 93, 403-444 (2009)

12. Gabbay, D.M., Schlechta, K.: An analysis of defeasible inheritance systems. Log. J. IGPL 17, 1-54 (2009)

13. Gabbay, D.M., Schlechta, K.: Reactive preferential structures and nonmonotonic consequence. Review of Symbolic Logic 2(2), 414-450 (2009)

14. Gabbay, D.M., Strasser, C.: Reactive standard deontic logic. J. Log. Comput. (to appear). Corner Deontic Logic

15. van Ditmarsch, H., van der Hoek, W., Kooi, B.: Dynamic Epistemic Logic. Springer, Berlin (2007) 